\title{
The Relations between Characterized Fuzzy Proximity, Fuzzy Compact, Fuzzy Uniform Spaces and Characterized Fuzzy $T_{s}-$ Spaces and Fuzzy $R_{k}$ - Spaces
}

\section{Abd-Allah AS $^{1}$ and Al-Khedhairi $\mathrm{A}^{2 *}$}

${ }^{1}$ Department of Mathematics, College of Science, El-Mansoura University, El-Mansoura, Egypt

${ }^{2}$ Department of Statistics and Operations Research, College of Science, King Saud University, PO Box 2455, Riyadh 11451, Saudi Arabia

\begin{abstract}
In this research work, we study the relations between the characterized fuzzy $T_{s}$-spaces and characterized fuzzy $R_{k}$-spaces presented in old papers, for $s \in\left\{0,1,2,3,3 \frac{1}{2}, 4\right\}$ and $k \in\left\{1,2,2 \frac{1}{2}, 3\right\}$ and the characterized fuzzy proximity spaces presented. We also study the relations between the characterized fuzzy $T_{s}$-spaces, the characterized fuzzy $R_{k}$-spaces and the characterized fuzzy compact spaces which is presented in old paper, as a generalization of the weaker and stronger forms of the G-compactness defined by Gähler. Moreover, we show here the relations between these characterized fuzzy $T_{s}$-spaces, characterized fuzzy $R_{k}$-spaces and the characterized fuzzy uniform spaces introduced and studied by Abd-Allah in 2013 as a generalization of the weaker and stronger forms of the fuzzy uniform spaces introduced by Gähler.
\end{abstract}

Keywords: Fuzzy filter; Fuzzy topological space; Operationsl; Isotone and idempotent; Characterized fuzzy space; $\varphi_{1,2}$-fuzzy neighborhood filters; Fuzzy uniform structure; Characterized fuzzy proximity space; Characterized fuzzy compact space; Characterized fuzzy uniform space; Characterized $F T_{s}$-space; $F \varphi_{1,2}-T_{s}$ space; Characterized $F R_{k}$-space and $F \varphi_{1,2}-R_{k}$ space for $s \in\left\{0,1,2,3,3 \frac{1}{2}, 4\right\} ; k \in\left\{1,2,2 \frac{1}{2}, 3\right\}$

\section{Introduction}

The notion of fuzzy filter has been introduced by Eklund et al. By means of this notion the point-based approach to fuzzy topology related to usual points has been developed. The more general concept for fuzzy filter introduced by Gähler [1] and fuzzy filters are classified by types. Because of the specific type of fuzzy filter however the approach of Eklund is related only to the fuzzy topologies which are stratified, that is, all constant fuzzy sets are open. The more specific fuzzy filters considered in the former papers are now called homogeneous. The operation on the ordinary topological space $(X, T)$ has been defined by Kasahara [2] as the mapping $\varphi$ from $T$ into $2^{X}$ such that $A \subseteq A^{\varphi}$, for all $A \in T$. In 1983, Abd El-Monsef et al. [3] extend Kasahara operation to the power set $P(X)$ of a set $X$. In 1999, Kandil [4] and the author extended Kasahars's and Abd El-Monsef's operations by introducing an operation on the class of all fuzzy subsets endowed with an fuzzy topology $\tau$ as the mapping $\varphi: L^{X} \rightarrow L^{X}$ such that int $\mu \leq \mu^{\varphi}$ for all $\mu \in L^{X}$, where $\mu^{\varphi}$ denotes the value of $\varphi$ at $\mu$.

The notions of the fuzzy filters and the operations on the class of all fuzzy subsets on $X$ endowed with a fuzzy topology $\tau$ are applied by Abd-Allah in [5-7] to introduce a more general theory including all the weaker and stronger forms of the fuzzy topology. By means of these notions the notion of $\varphi_{1,2}$-fuzzy interior of a fuzzy subset, $\varphi_{1,2}$-fuzzy convergence and $\varphi_{1,2}$-fuzzy neighborhood filters are defined and applied to introduced many special classes of separation axioms. The notion of $\varphi_{1,2}$-interior operator for a fuzzy subset is defined as a mapping $\varphi_{1,2}$.int: $L^{X}$ $\rightarrow L^{X}$ which fulfill (I1) to (I5) in Abd-Allah [5]. There is a one-to-one correspondence between the class of all $\varphi_{1,2}$-open fuzzy subsets of $X$ and these operators, that is, the class $\varphi_{1,2} O F(X)$ of all $\varphi_{1,2}$-open fuzzy subsets of $X$ can be characterized by these operators. Then the triple $\left(X, \varphi_{1,2}\right.$. int) as will as the triple $\left(X, \varphi_{1,2} \mathrm{OF}(X)\right)$ will be called the characterized fuzzy space [5] of $\varphi_{1,2}$-open fuzzy subsets. The characterized fuzzy spaces are identified by many of characterizing notions in Abd-Allah [5-7], for example by the $\varphi_{1,2}$-fuzzy neighborhood filters, $\varphi_{1,2}$-fuzzy interior of the fuzzy filters and by the set of $\varphi_{12}$-inner points of the fuzzy filters. Moreover, the notions of closeness and compactness in the characterized fuzzy spaces are introduced and studied by Abd-Allah in [7]. The notions of characterized $F T_{s}$-spaces, $\mathrm{F} \varphi_{1,2}-T_{s}$ spaces, characterized $F R_{k}$-spaces and $F \varphi_{1,2}-R_{k}$ spaces are introduced and studied in Abd-Allah [9-11] for all $s \in\left\{0,1,2,2 \frac{1}{2}, 3,3 \frac{1}{2}, 4\right\}$ and $k \in\left\{0,1,2,2 \frac{1}{2}, 3\right\}$. The notions of characterized fuzzy compact spaces, characterized fuzzy proximity spaces and characterized fuzzy uniform spaces are introduced and studied by the author in 2004 and 2013 in $[7,12]$. This paper is devoted to introduce and study the relations between the characterized $F T_{s}$ and $F R_{k}$-spaces, for $s \in\left\{0,1,2,3,3 \frac{1}{2}, 4\right\}$ and $k \in\left\{1,2,2 \frac{1}{2}, 3\right\}$, the characterized fuzzy proximity spaces and the characterized fuzzy compact spaces. Moreover, we show here the relations between these characterized $F T_{s}$ and $F R_{k}$-spaces and the characterized fuzzy uniform spaces. In section 2 , some definitions and notions related to the fuzzy subsets, fuzzy topologies, fuzzy filters, fuzzy proximity, operations on fuzzy subsets, $\varphi_{12}$-fuzzy neighborhood filters, characterized fuzzy space, characterized $F T_{s}$-spaces, $F \varphi_{1,2}-T_{s}$ spaces, characterized $F R_{k}$-spaces and $F \varphi_{1,2}-R_{k}$ spaces are given for $s \in\left\{0,1,2,3,3 \frac{1}{2}, 4\right\}$ and $k \in\left\{2,2 \frac{1}{2}, 3\right\}$. Section 3 , is devoted to

${ }^{*}$ Corresponding author: Al-Khedhairi A, Mathematics Department, College of Science and Humanity Studies, Prince Sattam Bin Abdul-Aziz University, PO Box 132012, Hotat Bani Tamim 11941, Saudi Arabia, Tel: 966800116 9528; E-mail: akhediri@ksu.edu.sa

Received December 08, 2016; Accepted December 31, 2016; Published January 26, 2017

Citation: Abd-Allah AS, Al-Khedhairi A (2017) The Relations between Characterized Fuzzy Proximity, Fuzzy Compact, Fuzzy Uniform Spaces and Characterized Fuzzy $T_{s}$-Spaces and Fuzzy $R_{k}$-Spaces. J Appl Computat Math 6: 337. doi: 10.4172/2168-9679.1000337

Copyright: (c) 2017 Abd-Allah AS, et al. This is an open-access article distributed under the terms of the Creative Commons Attribution License, which permits unrestricted use, distribution, and reproduction in any medium, provided the original author and source are credited. 
Citation: Abd-Allah AS, Al-Khedhairi A (2017) The Relations between Characterized Fuzzy Proximity, Fuzzy Compact, Fuzzy Uniform Spaces and Characterized Fuzzy $T_{s}$-Spaces and Fuzzy $R_{k}$-Spaces. J Appl Computat Math 6: 337. doi: 10.4172/2168-9679.1000337

Page 2 of 15

introduce and study the relation between the characterized fuzzy proximity spaces and our classes of the characterized $F T$-spaces and of the characterized $F R_{k}$-spaces. It will be shown that in the characterized fuzzy space $\left(X, \varphi_{1,2}\right.$. int $)$, the fuzzy proximity $\delta$ will be identified with the finer relation on the $\varphi_{12}$-fuzzy neighborhood filters. Also, we will show that any fuzzy proximity is separated if and only if the associated characterized fuzzy proximity space is characterized $F T_{0}$ and to each fuzzy proximity is associated a characterized $F R_{2}$-space in our sense. Generally, it will be shown that the associated characterized fuzzy proximity space $\left(X, \varphi_{1,2}\right.$. int $\left._{\delta}\right)$ is characterized $F R_{2}$-space if the related fuzzy topological space $(X, \tau)$ is $F \varphi_{1,2}-R_{2}$ space. Moreover, for each characterized $F R_{3}$-space the binary relation on $L^{X}$ defined by means the $\varphi_{1,2}$-fuzzy closure operator $\varphi_{1,2} . c l$ of $\tau$ in Equation (3.6), is fuzzy proximity on $X$ and conversely to each fuzzy proximity $\delta$ which has a $\varphi_{1,2}$-fuzzy closure operator fulfills the binary relation given in (3.6) is associated a characterized $F R_{3}$-space $\left(X, \varphi_{1,2} \cdot\right.$ int $\left._{\delta}\right)$. Moreover, when $L$ is a complete chain, $\varphi_{2} \geq 1_{{ }^{X}}$ is isotone and $\varphi_{1}$ is wfip with respect to $\varphi_{1}$ OF $(X)$, then we show that the associated characterized fuzzy space $\left(X, \varphi_{1,2}\right.$.int $)$ from the fuzzy normal topological space $(X, \tau)$ is finer than the associated characterized fuzzy proximity space $\left(X, \varphi_{1,2}\right.$.int $\left.{ }_{\delta}\right)$ by the fuzzy proximity $\delta$ defined by (3.6) and they identical if and only if $\left(X, \varphi_{1,2}\right.$.int $)$ is characterized $F T_{4}$-space. At the end of this section we prove that the associated characterized fuzzy proximity space $\left(X, \varphi_{1,2}\right.$. int $_{\delta}$ ) is characterized $F R_{2 \frac{1}{2}}$ - space and therefore it is characterized $F T_{3 \frac{1}{2}}$-space. There is a good notion of $\varphi_{1,2}$-fuzzy compactness of the fuzzy filters and of the fuzzy topological spaces introduced and studied by Abd-Allah et al. [7]. This notion fulfills many properties, for example, it fulfills the Tychonoff Theorem. In section 4, we used this notion to study the relations between the characterized fuzzy compact spaces and our classes of the characterized $F T$-spaces and of the characterized $F R_{k}$-spaces. It will be shown that every $\varphi_{1,2}$-closed subset of a characterized fuzzy compact space is $\varphi_{1,2}$-fuzzy compact and each $\varphi_{1,2}$-fuzzy compact subset of the characterized $F T_{2}$-space is $\varphi_{1,2}$-closed. Also, it will be shown that each characterized fuzzy compact $F T_{2}$-space is characterized $\mathrm{FT}_{4}$-space. Specially, we prove that the characterized fuzzy unit interval space $\left(I_{L}, \psi_{1,2} \cdot\right.$ int $\left._{T}\right)$ is characterized fuzzy compact $F T_{2}$-space and characterized $F T_{3 \frac{1}{1}}$-space. Generally, we show that every characterized fuzzy compact space is characterized $F T_{2}$-space if and only if it is characterized $F T_{3 \frac{1}{2}}$ - space. We show that, if $\left(X, \psi_{1,2}\right.$. int $)$ is characterized fuzzy compact space finer than the characterized $F T_{2}$-space $\left(X, \varphi_{1,2}\right.$.int $)$, then $\left(X, \varphi_{1,2}\right.$.int $\left.\tau\right)$ is $\varphi_{1,2} \psi_{1,2}$-fuzzy isomorphic to $\left(X, \psi_{1,2}\right.$.int $\left.{ }_{\sigma}\right)$. Moreover, if $\tau$ is finer than $\left.\sigma,\left(X, \varphi_{1,2} \text {.int }\right)_{\tau}\right)$ is characterized fuzzy compact space and $\left.\left(X, \psi_{1,2} \text {. int }\right)_{\sigma}\right)$ is characterized $F T_{3 \frac{1}{2}}$ - space, then $\left(X, \psi_{1,2}\right.$.int $)$ and $\left(X, \psi_{1,2}\right.$.int $\left.{ }_{\sigma}\right)$ are $\varphi_{1,2} \psi_{1,2}$-fuzzy isomorphic. The notion of fuzzy uniform structure had been introduced and studied by Gähler et al. [13]. This notion with the notion of the operations on the class of all fuzzy subsets are applied to introduce and study the notion of characterized fuzzy uniform spaces. In section 5 , we introduce and study the relations between the characterized fuzzy uniform spaces and our classes of the characterized $F T$-spaces and of the characterized $F R_{k}$ spaces. We show that the fuzzy uniform space $(X, \mathscr{U})$ is separated if and only if the associated characterized fuzzy uniform space $\left(X, \varphi_{1,2}\right.$.int $\left._{\varkappa}\right)$ is characterized $F T_{i}$-space but the fuzzy uniform space $(X, \mathcal{U})$ is separated if and only if the associated stratified fuzzy topological space $\left(X, \tau_{u}\right)$ is $F_{12}-T_{i}$ space for all $i \in\{0,1\}$. For each fuzzy uniform structure on a set $X$, we prove that there is an induced stratified fuzzy proximity on $L^{X}$. Moreover, both the fuzzy uniform structure and this induced stratified fuzzy proximity are associated with the same stratified characterized fuzzy uniform space. Finally, for each fuzzy uniform space $(X, \mathcal{U})$ we prove that the associated stratified characterized fuzzy uniform space

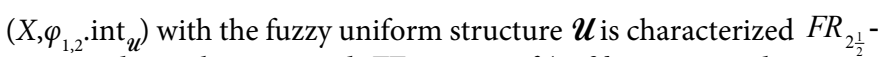
space and it is characterized $F T_{3 \frac{1}{2}}$ - space if $(X, \mathcal{U})$ is separated.

\section{Preliminaries}

We begin by recalling some facts on fuzzy subsets and on fuzzy filters. Let $L$ be a completely distributive complete lattice with different least and last elements 0 and 1, respectively. Let $L_{0}=L \backslash\{0\}$. Sometimes we will assume more specially that $L$ is a complete chain, that is, $L$ is a complete lattice whose partial ordering is a linear one. For a set $X$, let $L^{X}$ be the set of all fuzzy subsets of $X$, that is, of all mappings $f: X \rightarrow L$. Assume that an order-reversing involution $\alpha \mapsto \alpha^{\prime}$ of $L$ is fixed. For each fuzzy subset $\mu \in L^{X}$, let $\mu^{\prime}$ denote the complement of $\mu$ and it is given by the relation $\mu^{\prime}(x)=\mu(x)^{\prime}$ for all $x \in X$. Denote by $\bar{\alpha}$, the constant fuzzy subset of $X$ with value is $\alpha \in L$. For all $x \in X$ and for all $\alpha \in L_{0}$, the fuzzy subset $x_{\alpha}$ of $X$ whose value $\alpha$ at $x$ and 0 otherwise is called a fuzzy point in $X$. The set of all fuzzy points of a set $X$ will be denoted by $S(X)$.

\section{Fuzzy filters}

The fuzzy filter on $X[1]$ is the mapping $M: L^{X} \rightarrow L$ such that the following conditions are fulfilled:

(F1) $\boldsymbol{M}(\bar{\alpha}) \leq \alpha$ for all $\alpha \in L$ and $\boldsymbol{M}(\overline{1})=1$.

(F2) $\boldsymbol{M}(\mu \wedge \rho)=\boldsymbol{M}() \wedge \mathcal{M}(\rho)$ for all $\mu, \rho \in L^{X}$.

The fuzzy filter $\boldsymbol{M}$ is called homogeneous [14] if $\boldsymbol{M}(\bar{\alpha})=\alpha$ for all $\alpha \in L$. For each $x \in X$, the mapping $\dot{x}(\mu)=\mu(x)$ defined by $\dot{x}(\mu)=\mu(x)$ for all $\mu \in L^{X}$ is a homogeneous fuzzy filter on $X$. For each $\mu \in L^{X}$, the mapping $\dot{\mu}: L^{X} \rightarrow L$ defined by $\dot{\mu}(\eta)=\wedge \eta(x)$ for all $\eta \in L^{X}$ is a homogeneous fuzzy filter on $X$, called homogenous fuzzy filter at the fuzzy subset $\mu \in L^{X}$. Let $\mathscr{F}_{L} X$ and $\mathscr{F}_{L} X$ be the sets of all fuzzy filters and of all homogeneous fuzzy filters on $X$, respectively. If $\boldsymbol{M}$ and $\mathcal{N}$ are fuzzy filters on a set $X, \boldsymbol{M}$ is said to be finer than $\mathcal{N}$, denoted by $\boldsymbol{M}$ $\leq \mathcal{N}$, provided $\boldsymbol{M}(\mu) \geq \mathcal{N}(\mu)$ holds for all $\mu \in L^{X}$. Noting that if $L$ is a complete chain then $M$ is not finer than $N$, denoted by $\mathcal{M} \mathcal{N}$, provided there exists $\mu \in L^{X}$ such that $\boldsymbol{M}(\mu)<\mathcal{N}(\mu)$ holds.

\section{Lemma 2.1}

If $\mathcal{M}, \mathcal{N}$ and $\mathcal{L}$ are fuzzy filters on a set $X$. Then the following sentences are fulfilled [1].

$$
\boldsymbol{M} \neq \mathcal{L} \geq \mathcal{N} \text { implies } \boldsymbol{M} \neq \mathcal{N} \text { and } \boldsymbol{M} \geq \mathcal{L} \neq \mathcal{N} \text { implies } \boldsymbol{M} \neq \mathcal{N}
$$

\section{Proposition 2.1}

For all $\mu, \rho \in L^{X}$, we have $\mu \leq \rho$ if and only if $\dot{\mu} \leq \dot{\rho}$ [15].

For each non-empty set $\mathcal{A}$ of the fuzzy filters on $X$ the supremum $\bigvee_{\mathscr{M} \in \mathcal{A}} \mathcal{M}$ exists [1] and given by

$$
\left(\bigvee_{\mathcal{M} \in \mathcal{A}} \mathcal{M}\right)(\mu)=\bigwedge_{\mathcal{M} \in \mathcal{A}} \mathcal{M}(\mu)
$$

for all $\mu \in L^{X}$. Whereas the infimum $\bigwedge_{\mathscr{M} \in \mathcal{A}} \boldsymbol{M}$ of $A$ does not exists in general as an fuzzy filter. If the infimum $\bigwedge_{\mathscr{M} \in \mathcal{A}} \mathcal{M}$ exists, then we have

$$
(\widehat{\mathcal{M}} \in \mathcal{A} \mathcal{M})(\mu)=\bigvee_{\substack{\mu_{1} \cdots \cdots \mu_{n} \leq \mu, \mathcal{M}_{1}, \ldots, \mathcal{M}_{n} \in \mathcal{A}}}\left(\mathcal{M}_{1}\left(\mu_{1}\right) \wedge \cdots \wedge \mathcal{M}_{n}\left(\mu_{n}\right)\right),
$$

for all $\mu \in L^{X}$, where $n$ is an positive integer, $\mu_{1}, \ldots, \mu_{n}$ is a collection such that $\mu_{1} \wedge \ldots \wedge \mu_{n} \leq \mu$ and $\boldsymbol{M}_{1}, \ldots, \boldsymbol{M}_{n}$ are fuzzy filters from $\mathcal{A}$. Let $X$ be a set and $\mu \in L^{X}$, then the homogeneous fuzzy filter $\dot{\mu}$ at $\mu$ is the fuzzy filter on $X$ given by:

$$
\dot{\mu}=\underset{0<\mu(x)}{\bigvee} \dot{x},
$$


Citation: Abd-Allah AS, Al-Khedhairi A (2017) The Relations between Characterized Fuzzy Proximity, Fuzzy Compact, Fuzzy Uniform Spaces and Characterized Fuzzy $T_{s}$-Spaces and Fuzzy $R_{k}$-Spaces. J Appl Computat Math 6: 337. doi: 10.4172/2168-9679.1000337

Page 3 of 15

\section{Fuzzy filter bases}

The family $\left(\beta_{\alpha}\right) \alpha \in\left(\mathscr{B}_{\alpha}\right)_{\alpha \in L_{0}}$ of a non-empty subsets of $L^{X}$ is called a valued fuzzy filter base [1] if the following conditions are fulfilled:

(V1) $\in \beta_{\alpha}$ implies $\alpha \leq \sup \mu$.

(V2) For all $\alpha, \beta L_{0}$ with $\alpha \wedge \beta \in L_{0}$ and all $\in \beta_{\alpha}$ and $\rho \in \beta_{\beta}$ there are $\gamma \geq \alpha \wedge \beta$ and $\eta \leq \mu \rho$ such that $\eta \in \beta_{\gamma}$.

As shown in Gähler [1], each valued fuzzy filter base $\left(\mathscr{B}_{\alpha}\right)_{\alpha \in L_{0}}$ defines a fuzzy filter $\boldsymbol{M}$ on $X$ by $\boldsymbol{M}(\mu)=\underset{\rho \in \mathscr{B}_{\alpha}, \rho \leq \mu}{\vee} \alpha$ for all $\mu \in L^{X}$. Conversely, each fuzzy filter $M$ can be generated by a valued fuzzy filter base, e.g. by $(\alpha-\mathrm{p} r \boldsymbol{M})_{\alpha \in L_{0}}$ with $\alpha$-p $r \boldsymbol{M}=\left\{\mu \in L^{X} \mid \alpha \leq \mathcal{M}(\mu)\right\}$. The $(\alpha-\mathrm{p} r \boldsymbol{M})_{\alpha \in L_{0}}$ is the family of pre filters on $X$ and it is called the large valued fuzzy filter base of $\boldsymbol{M}$. Recall that the pre filter on $X[16]$ is a non-empty proper subset $F$ of $L^{X}$ such that (1) $\mu, \rho \in F$ implies $\mu \wedge \rho \in F$ and (2) from $\mu \in F$ and $\mu \leq$ it follows $\rho \in F$.

\section{Valued and superior principal fuzzy filters}

Let a non-empty set $X$ be fixed, $\mu \in L^{X}$ and $\alpha \in L$ such that $\alpha \leq \sup$ $\mu$, the valued principal fuzzy filter [20] generated by $\mu$ and $\alpha$, will be denoted by $[\mu, \alpha]$, is the fuzzy filter on $X$ which has $\left(\mathscr{B}_{\beta}\right)_{\beta \in L_{0}}$ with $\beta_{\beta}=\{\mu\}$ if $0 \leq \beta \leq \alpha$ and $\mathscr{B}_{\beta}=\{1\}$ otherwise as a valued fuzzy filter base. For all $\eta \in L^{X}$, we have $[\mu, \alpha](\eta)=0$ if $\mu \not \leq \eta,[\mu, \alpha](\eta)=\alpha$ if $\mu \leq \eta \neq \overline{1}$ and $[\mu, \alpha](\eta)=1$ if $\eta=\overline{1}$. Moreover, for each $\beta \in L_{0}$ we have $\beta$-pr $[\mu, \alpha]=\{\eta \mid$ $\mu \leq \eta\}$ if $\beta \leq \alpha$ and $\beta-\operatorname{pr}[\mu, \alpha]=\{\overline{1}\}$ otherwise. The superior principal fuzzy filter [1] generated by $\mu$, written $[\mu]$, is the homogeneous fuzzy filter on $X$ which has $\beta=\{\mu \wedge \bar{\alpha} \mid \alpha \in L\} \cup\{\bar{\alpha} \mid \alpha \in L\}$ as a superior fuzzy filter base. As shown in Katsaras [18], the superior principal fuzzy filter $[\mu]$ is representable by a fuzzy pre filter if and only if $\sup \mu=1$.

\section{Fuzzy filter functors and fuzzy filter monads}

The fuzzy filter functor $\mathcal{F}_{L}: S E T \rightarrow S E T$ is the covariant functor from the category SET of all sets to this category which assigns to each set $X$ the set $\mathscr{F}_{L} X$ and to each mapping $f: X^{\circledast} Y$ the mapping $\mathscr{F}_{L} f: \mathscr{F}_{L} X \rightarrow \mathscr{F}_{L} Y$. The homogeneous fuzzy filter functor $F_{L}: S E T \rightarrow S E T$ is the sub fuzzy filter functor of $F_{L}$ which assigns to each set $X$ the set $F_{L} X$ and to each mapping $f$ $: X^{\oplus} Y$ the domain-range restriction $F_{L} f: F_{L} X \rightarrow F_{L} Y$ of the mapping $\mathcal{F}_{L} f: \mathcal{F}_{L} X$ $\rightarrow \mathscr{F}_{L} Y$. For each set $X$, let $\eta_{X}: X \rightarrow \mathscr{F}_{L} X$ be the mapping defined by $\eta_{X}(x)=\dot{\mathrm{x}}$ for all $x \in X$, and let $e_{X}: L^{X} \rightarrow L^{\mathscr{F}_{\mathcal{L}} X}$ be the mapping for which $e_{X}(\mu)(M)$ $=M(\mu)$ for all $\mu L^{X}$ and $M \in F_{L} X$. Moreover, let $\mu_{X}: \mathscr{F}_{L}\left(\mathscr{F}_{L} X\right) \rightarrow \mathscr{F}_{L} X$ be the mapping which assigns to each fuzzy filter $\mathcal{L}_{\text {on }} \mathscr{F}_{L} X$ the fuzzy filter $\mu_{X}(\mathcal{L})=\mathcal{L} \circ e_{X}$ on $X . \quad \eta=\left(\eta_{X}\right)_{X \in \mathrm{Ob}(S E T)}:$ id $\rightarrow \mathscr{F}_{L}$ with id the identity set functor and $\mu=\left(\mu_{X}\right)_{X \in \mathrm{Ob}(S E T)}: \mathscr{F}_{L} \circ \mathscr{F}_{L} \rightarrow \mathscr{F}_{L}$ are natural transformations. $\left(\mathcal{F}_{L}, \eta, \mu\right)$ is a monad in the categorical sense, called the fuzzy filter monad [1], that is, $\mu_{X} \circ \mathscr{F}_{L}\left(\eta_{X}\right)=\mu_{X} \circ \eta_{\tilde{L}_{L} X}=1_{\mathscr{F}_{L} X}$ and $\mu_{X} \circ \mathscr{F}_{L}\left(\mu_{X}\right)=\mu_{X} \circ \mu_{\mathscr{F}_{X}}$ for each set $X$. Related to the sub functor $F_{L}$ of $\mathcal{F}_{L}$, there are analogous natural transformations as $\eta$ and $\mu$, denoted $\eta^{\prime}$ and $\mu^{\prime}$, respectively. $\eta^{\prime}$ consists of the rangerestrictions $\eta_{X}^{\prime}: X \rightarrow F_{L} X$ of the mappings $\eta_{X^{*}} \mu^{\prime}$ is the family of all mappings $\mu_{X}^{\prime}: F_{L}\left(F_{L} X\right) \rightarrow F_{L} X$ defined by $\mu_{X}^{\prime}(\mathcal{L})=\mathcal{L} \circ e_{X}^{\prime}$ for all homogeneous fuzzy filters $L$ on $F_{L} X$, where $e_{X}^{\prime}: L^{X} \rightarrow L^{F_{L} X}$ is the mapping given by $e_{X}^{\prime}(\mu)(\boldsymbol{M})=\boldsymbol{M}(\mu)$ for all $\mu \in L^{X}$ and $\boldsymbol{M} \in F_{L} X$. As has been shown in Gähler et al. [13], $\left(F_{L}, \eta^{\prime}, \mu^{\prime}\right)$ is a sub monad of $\left(F_{L}, \eta, \mu\right)$ $\left(\mathscr{F}_{L}, \eta, \mu\right)$, that is, for the inclusion mappings $i_{X}: F_{L} X \rightarrow \mathscr{F}_{L} X$ we have $\eta_{X}=i_{X} \circ \eta_{X}^{\prime}$ and $\mu_{X} \circ \mathscr{F}_{L} i_{X} \circ i_{F_{L} X}=i_{X} \circ \mu_{X}^{\prime}$ for all sets $X$.

\section{Fuzzy topologies}

By a fuzzy topology on a set $X[20,21]$, we mean a subset of $L^{X}$ which is closed with respect to all suprema and all finite infima and contains the constant fuzzy sets $\overline{0}$ and $\overline{1}$. A set $X$ equipped with an fuzzy topology $\tau$ on $X$ is called fuzzy topological space. For each fuzzy topological space $(X, \tau)$, the elements of $\tau$ are called open fuzzy subsets of this space. If $\tau_{1}$ and $\tau_{2}$ are two fuzzy topologies on a set $X$, then $\tau_{2}$ is said to be finer than $\tau_{1}$ and $\tau_{1}$ is said to be coarser than $\tau_{2}$, provided $\tau_{1} \subseteq \tau_{2}$ holds. The fuzzy topological space $(X, \tau)$ and also $\tau$ are said to be stratified provided $\bar{\alpha} \in$ holds for all $\alpha \in L$, that is, all constant fuzzy subsets are open [17].

\section{Fuzzy proximity spaces}

The binary relation $\delta$ on $L^{X}$ is called fuzzy proximity on $X$ [18], provided it fulfill the following conditions: of $\delta$.

(P1) $\mu \bar{\delta} \rho$ implies $\rho \bar{\delta} \mu$ for all $\mu, \rho \in L^{X}$, where $\bar{\delta}$ is the negation

(P2) $(\mu \vee \rho) \bar{\delta} \eta$ if and only if $\mu \bar{\delta} \eta$ and $\rho \bar{\delta} \eta$ for all $\mu, \rho, \eta \in L^{X}$.

(P3) $\mu=\overline{0}$ or $\rho=\overline{0}$ implies $\mu \bar{\delta} \rho$ for all $\mu, \rho \in L^{X}$.

(P4) $\mu \bar{\delta} \rho$ implies $\mu \leq \rho^{\prime}$ for all $\mu, \rho \in L^{X}$.

(P5) If $\mu \bar{\delta} \rho$, then there is an $\eta \in L^{X}$ such that $\mu \bar{\delta} \eta$ and $\eta^{\prime} \bar{\delta} \rho$.

The set $X$ equipped with an fuzzy proximity $\delta$ on $X$ is said to be fuzzy proximity space and will be denoted by $(X, \delta)$. Every fuzzy proximity $\delta$ on a set $X$ is associated an fuzzy topology on $X$ denoted by $\tau_{\delta}$. The fuzzy proximity $\delta$ on a set $X$ is said to be separated if and only if for all $x, y \in X$ such that $x \neq y$ we have $x_{\alpha} \bar{\delta} y_{\beta}$ for all $\alpha, \beta \in L_{0}$.

\section{Operation on fuzzy sets}

In the sequel, let a fuzzy topological space $(X, \tau)$ be fixed. By the operation [4] on a set $X$, we mean the mapping $\varphi: L^{X} \rightarrow L^{X}$ such that int $\mu \leq \mu^{\varphi}$ holds, for all $\mu \in L^{X}$, where $\mu^{\varphi}$ denotes the value of $\varphi$ at $\mu$. The class of all operations on $X$ will be denoted by $O_{\left(L^{X}, \tau\right)}$. By the identity operation on $O_{\left(L^{X}, \tau\right)}$, we mean the operation $1_{L^{X}}: L^{X} \rightarrow L^{X}$ such that $1_{L^{X}}(\mu)=\mu$ for all $\mu \in L^{X}$. Also by the constant operation on $O_{\left(L^{X}, \tau\right)}$, we mean the operation $c_{L^{X}}: L^{X} \rightarrow L^{X}$ such that $c_{L^{X}}(\mu)=\overline{1}$ for all $\mu \in L^{X}$. If $\leq$ is a partially ordered relation on $O_{\left(L^{X}, \tau\right)}$ defined as by $\varphi_{1} \leq \varphi_{2} \Leftrightarrow \mu^{\varphi_{1}} \leq \mu^{\varphi_{2}}$ for all $\mu \in L^{X}$, then obviously, $\left(O_{\left(L^{X}, \tau\right)}, \leq\right)$ is a completely distributive lattice. As an application on this partially ordered relation, the operation $\varphi: L^{X} \rightarrow L^{X}$ will be called:

(i) Isotone if $\mu \leq \rho$ implies $\mu^{\varphi} \leq \rho^{\varphi}$ holds, for all $\mu, \rho \in L^{X}$.

(ii) Weakly finite intersection preserving (wfip, for short) with respect to $\mathcal{A} \subseteq L^{X}$ if $\rho \wedge \mu^{\varphi} \leq(\rho \wedge \mu)^{\varphi}$ holds, for all $\rho \in A$ and $\mu \in L^{X}$.

(iii) Idempotent if $\mu^{\varphi}=\left(\mu^{\varphi}\right)$, for all $\mu \in L^{X}$.

The operations $\varphi, \psi \in O_{\left(L^{X}, \tau\right)}$ are said to be dual if $\mu=\operatorname{co}((\operatorname{co\mu } \mu))$ or equivalently $\varphi \mu=c o(\psi(c o \mu))^{\tau)}$ for all $\mu \in L^{X}$, where co $\mu$ denotes the complement of $\mu$. The dual operation of $\varphi$ is denoted by $\tilde{\varphi}$. In the classical case of $\mathrm{L}=\{0,1\}$, by the operation on the set $X$ [3], we mean the mapping $\varphi: P(X) \rightarrow P(X)$ such that int $A \leq A^{\varphi}$ for all $A$ in the power set $P(X)$ and the identity operation on the class of all ordinary operations $O_{(P(X), T)}$ on $X$ will be denoted by $i_{P(X)}$, where $i_{P(X)}(A)=A$ for all $A \in P(X)$. 
Citation: Abd-Allah AS, Al-Khedhairi A (2017) The Relations between Characterized Fuzzy Proximity, Fuzzy Compact, Fuzzy Uniform Spaces and Characterized Fuzzy $T_{s}$-Spaces and Fuzzy $R_{k}$-Spaces. J Appl Computat Math 6: 337. doi: 10.4172/2168-9679.1000337

Page 4 of 15

\section{$\varphi$-open fuzzy subsets}

Let a fuzzy topological space $(X, \tau)$ be fixed and $\varphi \in O_{\left(L^{X}, \tau\right)}$. The fuzzy subset $\mu: X \rightarrow L$ is said to be $\varphi$-open fuzzy subset if $\mu \leq \mu^{\varphi}$ holds. We will denote the class of all $\varphi$-open fuzzy subsets on $X$ by OF $(X)$. The fuzzy subset $\mu$ is called $\varphi$-closed if its complement $\operatorname{co} \mu$ is $\varphi$-open. The two operations $\varphi, \psi \in O_{\left(L^{X}, \tau\right)}$ are equivalent and written $\varphi \sim \psi$ if and only if $\varphi$ OF $(X)=\psi \mathrm{OF}(X)$.

\section{$\varphi_{1,2}$-interior of fuzzy subsets}

Let a fuzzy topological space $(X, \tau)$ be fixed and $\varphi_{1}, \varphi_{2} \in O_{\left(L^{X}, \tau\right)}$. Then the $\varphi_{1,2}$-interior of the fuzzy subset $\mu: X \rightarrow L$ is the mapping $\varphi_{1,2}$. int $\mu: X \rightarrow L$ defined by:

$$
\varphi_{1,2} \text {.int } \mu=\underset{\rho \in \varphi_{1} O F(X), \rho^{\varphi_{2} \leq \mu}}{\vee} \rho,
$$

As easily seen that $\varphi_{12}$.int $\mu$ is the greatest $\varphi_{1}$-open fuzzy subset $\rho$ such that $\rho^{\varphi_{2}}$ less than or equal to $\mu$ [5]. The fuzzy subset $\mu$ is said to be $\varphi_{1,2}$-open if $\mu \leq \varphi_{1,2}$.int $\mu$. The class of all $\varphi_{1,2}$-open fuzzy subsets of $X$ will be denoted by $\varphi_{1,2} \operatorname{OF}(X)$. The complement $\operatorname{co\mu }$ of a $\varphi_{1,2}$-open fuzzy subset $\mu$ will be called $\varphi_{1,2}$-closed and the class of all $\varphi_{1,2}$-closed fuzzy subsets of $X$ will be denoted by $\varphi_{1,2} C F(X)$. In the classical case of $L=\{0,1\}$, we note that the fuzzy topological space $(X, \tau)$ is up to an identification by the ordinary topological space $(X, T)$ and $\varphi_{1,2}$.int $\mu$ is the classical one. Hence, in this case the ordinary subset $A$ of $X$ is $\varphi_{1,2^{-}}$ open if $\mathrm{A} \subseteq \varphi_{1,2}$.int $A$. The complement of the $\varphi_{1,2}$-open subset $A$ of $X$ will be called $\varphi_{1,2}$-closed. The class of all $\varphi_{1,2}$-open and the class of all $\varphi_{1,2}$-closed subsets of $X$ will be denoted by $\varphi_{1,2} O(X)$ and $\varphi_{1,2} C(X)$, respectively. Clearly, $F$ is $\varphi_{1,2}$-closed if and only if $\varphi_{1,2} \cdot \mathrm{cl}_{\mathrm{T}} F=F$.

\section{Proposition 2.2}

If $(X, \tau)$ is an fuzzy topological space and $\varphi_{1}, \varphi_{2} \in O_{\left(L^{X}, \tau\right)}$. Then, the mapping $\varphi_{1,2}$.int $\mu: X \rightarrow L$ fulfills the following axioms [5]:

(i) If $\varphi_{2} \geq 1_{L^{X}}$, then $\varphi_{1,2}$.int $\mu \leq \mu$ holds.

(ii) $\varphi_{1,2}$.int is isotone operator, that is, if $\mu \leq \rho$ then, ${ }_{1,2}$.int $\mu \leq \varphi_{1,2}$.int $\rho$ holds for all $\mu, \rho \in L^{X}$.

(iii) $\varphi_{1,2} \cdot \operatorname{int} \overline{1}=\overline{1}$.

(iv) If $\varphi_{2} \geq 1_{L^{X}}$ is isotone and $\varphi_{1}$ is wfip with respect to $\varphi_{1} \mathrm{OF}(X)$, then ${ }_{1,2}$.int $(\mu \wedge \rho)=\varphi_{1,2}$.int $\mu \wedge \varphi_{1,2}$.int $\rho$ for all $\mu, \rho \in L^{X}$.

(v) If ${ }_{2}$ is isotone and idempotent operation, then $\varphi_{1,2}$.int $\mu \leq \varphi_{1,2}$. int $\left(\varphi_{1,2}\right.$.int $\left.\mu\right)$ holds.

(vi) $\varphi_{1,2} \cdot \operatorname{int}\left(\bigvee_{i \in I} \mu_{i}\right)=\bigvee_{i \in I} \varphi_{1,2}$.int $\mu_{i}$ for all $\mu_{i} \in \varphi_{1,2}$ OF $(X)$.

\section{Proposition 2.3}

Let $(X, \tau)$ be an fuzzy topological space and $\varphi_{1}, \varphi_{2} \in O_{\left(L^{X}, \tau\right)}$. Then the following are fulfilled [5]:

(i) If $\varphi_{2} \geq 1_{L^{X}}$, then the class $\varphi_{1,2} O F(X)$ forms extended fuzzy topology on $X[19]$.

(ii) If $\varphi_{2} \geq 1_{L^{X}}$ and $\varphi_{1,2} \cdot \operatorname{int} \overline{1}=\overline{1}$, then the class $\varphi_{1,2} O F(X)$ forms a supra fuzzy topology on $X$ [19].

(iii) If $\varphi_{2} \geq 1_{L^{X}}$ is isotone and $\varphi_{1}$ is wfip with respect to $\varphi_{1} O F(X)$, then $\varphi_{1,2} O F(X)$ is fuzzy pre topology on $X$ [19].

(iv) If $\varphi_{2} \geq 1_{L^{X}}$ is isotone and idempotent operation and $\varphi_{1}$ is wfip with respect to $\varphi_{1} O F(X)$, then $\varphi_{1,2} O F(X)$ is a fuzzy topology on
$X[20,21]$.

From Propositions 2.2 and 2.3, if the fuzzy topological space $(X, \tau)$ be fixed and $\varphi_{1}, \varphi_{2} \in O_{\left(L^{X}, \tau\right)}$. Then

$\varphi_{1,2} O F(X)=\left\{\mu \in L^{X} \mid \mu \leq \varphi_{1,2} \cdot \operatorname{int} \mu\right\}$,

and the following conditions are fulfilled:

(I1) If $\varphi_{2} \geq 1_{L^{X}}$, then $\varphi_{1,2}$.int $\mu \leq \mu$ holds, for all $\mu \in L^{X}$.

(I2) If $\mu \leq \rho$, then $\varphi_{1,2}$.int $\mu \leq \varphi_{1,2}$.int $\rho$ for all $\mu, \rho \in L^{X}$.

(I3) $\varphi_{1,2} \cdot$ int $\overline{1}=\overline{1}$.

(I4) If $\varphi_{2} \geq 1_{L^{X}}$ is isotone and $\varphi_{1}$ is wfip with respect to $\varphi_{1} O F(X)$, then $\varphi_{1,2}$.int $\mu \wedge \varphi_{1,2}$.int $\rho=\varphi_{1,2}$.int $(\mu \wedge \rho)$ for all $\mu, \rho \in L^{X}$.

(I5) If ${ }_{1}$ is isotone and idempotent, then $\varphi_{1,2}$.int $\left(\varphi_{1,2}\right.$.int $\left.\mu\right)=\varphi_{1,2}$.int $\mu$ for all $\mu \in L^{X}$.

\section{Characterized fuzzy spaces}

Independently on the fuzzy topologies, the notion of $\varphi_{1,2}$-interior operator for fuzzy subsets can be defined as a mapping $\varphi_{1,2}$.int : $L^{X} \rightarrow$ $L^{X}$ which fulfill (I1) to (I5). It is well-known that (2.2) and (2.3) give a one-to-one correspondence between the class of all $\varphi_{1,2}$-open fuzzy subsets and these operators, that is, $\varphi_{1,2} O F(X)$ can be characterized by the $\varphi_{1,2}$-interior operators. In this case the triple $\left(X, \varphi_{1,2}\right.$.int $)$ as will as the triple $\left(X, \varphi_{1,2} O F(X)\right)$ will be called characterized fuzzy space [5] of the $\varphi_{1,2}$-open fuzzy subsets of $X$. For each characterized fuzzy space $\left(X, \varphi_{1,2}\right.$ int $)$, the elements of $\varphi_{1,2}$ of $(X)$ are called $\varphi_{1,2}$-open fuzzy subsets of this space. If $\left(X, \varphi_{1,2}\right.$.int $)$ and $\left(X, \psi_{1,2}\right.$.int $)$ are two characterized fuzzy spaces, then $\left(X, \varphi_{1,2}\right.$.int $)$ is said to be finer than $\left(X, \psi_{1,2}\right.$.int $)$ and denoted by $\varphi_{1,2}$.int $\leq \psi_{1,2}$.int provided $\varphi_{1,2}$.int $\mu \geq \psi_{1,2}$.int $\mu$ holds for all $\mu \in L^{X}$. The characterized fuzzy space $\left(X, \varphi_{1,2}\right.$.int $)$ is said to be stratified if and only if $\varphi_{1,2}$.int $\bar{\alpha}=\bar{\alpha}$ for all $\alpha \in L$. As shown in Abd-Allah [5], the characterized fuzzy space $\left(X, \varphi_{1,2}\right.$.int $)$ is stratified if the related fuzzy topology is stratified. Moreover, the characterized fuzzy space $\left(X, \varphi_{1,2}\right.$.int $)$ is said to have the weak infimum property [19] provided $\varphi_{1,2} \cdot \operatorname{int}(\mu \wedge \bar{\alpha})=\varphi_{1,2}$. int $\mu \wedge \varphi_{1,2}$.int $\bar{\alpha}$ for all $\mu \in L^{X}$ and $\alpha \in L$. The characterized fuzzy space $\left(X, \varphi_{1,2}\right.$.int) is said to be strongly stratified provided $\varphi_{1,2}$.int is stratified and have the weak infimum property.

\section{Fuzzy unit interval}

The fuzzy unit interval will be denoted by $I_{L}$ and it is defined in Gähler [24] as the fuzzy subset $I_{L}=\left\{x \in \mathbb{R}_{\mathrm{L}}^{*} \mid x \leq 1^{\sim}\right\}$, where $I=[0,1]$ is the real unit interval and $\mathbb{R}_{\mathrm{L}}^{\star}=\left\{x \in \mathbb{R}_{\mathrm{L}} \mid x(0)=1\right.$ and $\left.0^{\sim} \leq x\right\}$ is the set of all positive fuzzy real numbers. Note that, the binary relation $\leq$ is defined on $\mathbb{R}_{\mathrm{L}}$ as follows: $x \leq y \Leftrightarrow x_{\alpha_{1}} \leq y_{\alpha_{1}}$ and $x_{\alpha_{2}} \leq y_{\alpha_{2}}$, for all $x, y \in \mathbb{R}_{\mathrm{L}}$, where $x_{\alpha_{1}}=\inf \{z \in \mathbb{R} \mid x(z) \geq \alpha\}$ and $x_{\alpha_{2}}=\sup \{z \in \mathbb{R} \mid x(z) \geq \alpha\}$ for all $\alpha \in L_{0}$. Note that the family $\Omega$ which is defined by: $\Omega=\left\{R_{\delta}\left|I_{L}\right| \delta \in I\right\} \cup\left\{R^{\delta}\left|I_{L}\right| \quad \delta \in I\right\} \cup\left\{0^{\sim} \mid I_{L}\right\}$ is a base for a fuzzy topology $I$ on $I_{L}$ and the order pair $\left(I_{L}, I\right)$ is said to be fuzzy unit interval topological space, where $R_{\delta}$ and $R^{\delta}$ are the fuzzy subsets of $\mathbb{R}_{\mathrm{L}}$ defined by $R_{\delta}(x)=\bigvee_{\alpha>\delta} x(\alpha)$ and $R^{\delta}(x)=\left(\bigvee_{\alpha \geq \delta} x(\alpha)\right)^{\prime}$ for all $x \in \mathbb{R}_{\mathrm{L}}$ and $\delta \in \mathbb{R}$. IThe restrictions of $R_{\delta}$ and $R^{\delta}$ on $I_{L}$ are the fuzzy subsets $R^{\delta} \mid I_{L}$ and $R^{\delta} \mid I_{L}$, respectively. Recall that the inequality $R^{\delta}(x) \wedge$ $R^{\gamma}(y) \leq R^{\delta+\gamma}(x+y)$ holds, where $x+y$ is the fuzzy real number defined by: $(x+y)(\xi)=\underset{\gamma, \zeta \in \mathbb{R}, \gamma+\zeta=\xi}{\bigvee}(x(\gamma) \wedge y(\zeta))$ for all $\xi \in \mathbb{R}$. Consider a fuzzy unit interval topological space $\left(I_{I}, I\right)$ be given and $\psi_{1}, \psi_{2} \in O_{\left(I_{L}, \Im\right)}$, then in this work the characterized fuzzy space $\left.\left(I_{L}, \psi_{1,2} \text {.int }\right)_{I}\right)$ will be called 
Citation: Abd-Allah AS, Al-Khedhairi A (2017) The Relations between Characterized Fuzzy Proximity, Fuzzy Compact, Fuzzy Uniform Spaces and Characterized Fuzzy $T_{s}$-Spaces and Fuzzy $R_{k}$-Spaces. J Appl Computat Math 6: 337. doi: 10.4172/2168-9679.1000337

Page 5 of 15

characterized fuzzy unit interval space and we define the cartesian product of a number of copies of the fuzzy unit interval $I_{L}$ equipped with the product of the characterized fuzzy unit interval spaces generated by $\psi_{1,2}$. int $_{\mathrm{I}}$ on it as a characterized fuzzy cube.

\section{$\varphi_{1,2}$-fuzzy neighborhood filters}

An important notion in the characterized fuzzy space $\left(X, \varphi_{1,2}\right.$.int $)$ is that of the $\varphi_{12}$-fuzzy neighborhood filter at the point and at the ordinary subset in this space. Let $(X, \tau)$ be a fuzzy topological space and $\varphi_{1}, \varphi_{2} \in O_{\left(L^{X}, \tau\right)}$. As follows by (I1) to (I5) for each $x \in X$, the mapping $\mathcal{N}_{\varphi_{1,2}}(x): L^{X} \rightarrow L$ which is defined by:

$$
\mathcal{N}_{\varphi_{1,2}}(x)(\mu)=\left(\varphi_{1,2} \text {.int } \mu\right)(x),
$$

for all $\mu \in L^{X}$ is a fuzzy filter, called $\varphi_{1,2}$-fuzzy neighborhood filter at $x$ [5]. If $\varnothing \neq \mathrm{F} \in P(X)$, then the $\varphi_{1,2}$-fuzzy neighborhood filter at $\mathrm{F}$ will be denoted by $\mathcal{N}_{\varphi_{1,2}}(\mathrm{~F})$ and it will be defined by:

$$
\mathcal{N}_{\varphi_{1,2}}(\mathrm{~F})=\bigvee_{x \in \mathrm{F}} \mathcal{N}_{\varphi_{1,2}}(x) \text {. }
$$

Since $\mathcal{N}_{\varphi_{1,2}}(x)$ is fuzzy filter for all $x \in X$, then $\mathcal{N}_{\varphi_{1,2}}(\mathrm{~F})$ is also fuzzy filter on $X$. Because of $\left[\chi_{\mathrm{F}}\right]=\bigvee_{x \in \mathrm{F}} \dot{x}$, then we have $\mathcal{N}_{\varphi_{1,2}}(\mathrm{~F}) \geq\left[\chi_{\mathrm{F}}\right]$ holds. If the related $\varphi_{1,2}$-interior operator fulfill the axioms (I1) and (I2) only, then the mapping $\mathscr{N}_{\varphi_{1,2}}(x): L^{X} \rightarrow L$, defined by (2.4) is an fuzzy stack, called $\varphi_{1,2}$-fuzzy neighborhood stack at $x$. Moreover, if the $\varphi_{1,2}$ interior operator fulfill the axioms (I1), (I2) and (I4) such that in (I4) instead of $\rho \in L^{X}$ we take $\bar{\alpha}$, then the mapping $\mathcal{N}_{\varphi_{1,2}}(x): L^{X} \rightarrow L$, is an fuzzy stack with the cutting property, called $\varphi_{1,2}$-fuzzy neighborhood stack with the cutting property at $x$. Obviously, the $\varphi_{1,2}$-fuzzy neighborhood filters fulfill the following conditions:

(N1) $\dot{x} \leq \mathscr{N}_{\varphi_{1,2}}(x)$ holds for all $x \in X$.

(N2) $\mathscr{N}_{\varphi_{1,2}}(x)(\mu) \leq \mathcal{N}_{\varphi_{1,2}}(x)(\rho)$ holds for all $\mu, \rho \in L^{X}$ and $\mu \leq \rho$. $\mu \in L^{X}$

(N3) $\mathscr{N}_{\varphi_{1,2}}(x)\left(y \mapsto \mathscr{N}_{\varphi_{1,2}}(y)(\mu)\right)=\mathscr{N}_{\varphi_{1,2}}(x)(\mu)$, for all $x \in X$ and

Clearly, $y \mapsto \mathcal{N}_{\varphi_{1,2}}(y)(\mu)$ is the fuzzy subset $\varphi_{1,2}$.int $\mu$.

The characterized fuzzy space $\left(X, \varphi_{1,2}\right.$.int $)$ is characterized as the fuzzy filter pre topology [5], that is, as a mapping $\mathcal{N}_{\varphi_{1,2}}: X \rightarrow \mathscr{F}_{L} X$ such that the conditions (N1) to (N3) are fulfilled.

\section{$\varphi_{1,2} \psi_{1,2}-$ fuzzy continuity}

Let now the fuzzy topological spaces $\left(X, \tau_{1}\right)$ and $\left(Y, \tau_{2}\right)$ are fixed, $\varphi_{1}, \varphi_{2} \in O_{\left(L^{X}, \tau_{1}\right)}$ and $\psi_{1}, \psi_{2} \in O_{\left(L^{Y}, \tau_{2}\right)}$. The mapping $f:\left(X, \varphi_{1,2}\right.$.int $) \rightarrow$ $\left(Y, \psi_{1,2}\right.$.int) is said to be $\varphi_{1,2} \psi_{1,2}-$ fuzzy continuous [5] if the inequality

$$
\left(\psi_{1,2} \cdot \operatorname{int} \eta\right) \circ f \leq \varphi_{1,2} \cdot \operatorname{int}(\eta \circ f),
$$

holds for all $\eta \in L^{Y}$. If an order reversing involution ' of $L$ is given, then we have that $f$ is a fuzzy continuous if and only if $\varphi_{1,2} \cdot \operatorname{cl}(\eta \circ f) \leq\left(\psi_{1,2} \cdot \operatorname{cl} \eta\right) \circ f$ holds for all $\eta \in L^{Y}$. Note that $\varphi_{1,2} . c \mathrm{cl}$ and $\psi_{1,2}$.cl, means that the closure operators related to $\varphi_{1,2}$.int and $\psi_{1,2}$.int, respectively which are defined by $\varphi_{1,2} . c l \mu=c o\left(\varphi_{1,2}\right.$.int $\left.\operatorname{co} \mu\right)$ for all $\mu$ $\in \in L^{X}$. Obviously if $f$ is $\varphi_{1,2} \psi_{1,2}$-fuzzy continuous and the inverse $f^{-1}$ of $f$ exists, then $f^{1}:\left(Y, \psi_{1,2}\right.$.int $) \rightarrow\left(X, \varphi_{1,2}\right.$.int $)$ is $\psi_{1,2} \varphi_{1,2}$-fuzzy continuous, that is, $\left(\varphi_{1,2}\right.$.int $\left.\mu\right) \circ f^{-1} \leq \psi_{1,2} \cdot \operatorname{int}\left(\mu \circ f^{-1}\right)$ holds for all $\mu \in L^{X}$. By means of characterizing the $\varphi_{1,2}$-fuzzy neighborhoods $\mathcal{N}_{\varphi_{1,2}}(x)$ of $\varphi_{1,2}$.int and
$\mathcal{N}_{\psi_{1,2}}(x)$ of $\psi_{1,2}$.int which are defined by (2.4), the fuzzy continuity of $f$ can also be characterized as follows:

The mapping $f:\left(X, \varphi_{1,2}\right.$.int $) \rightarrow\left(Y, \psi_{1,2}\right.$.int $)$ is $\varphi_{1,2} \psi_{1,2}$-fuzzy continuous if the inequality $\mathscr{N}_{\psi_{1,2}}(f(x)) \geq \mathscr{F}_{L} f\left(\mathscr{N}_{\varphi_{1,2}}(x)\right)$ holds for each $x \in X$. Obviously, in case of $L=\{0,1\}, \varphi_{1}=\psi_{2}=$ int, $\varphi_{2}=1_{L^{X}}$ and $\psi_{2}=1_{L^{Y}}$, the $\varphi_{1,2} \psi_{1,2}$-fuzzy continuity coincides with the usual fuzzy continuity.

\section{$\varphi_{1,2}$-fuzzy convergence}

Let an fuzzy topological space $(X, \tau)$ be fixed and $\varphi_{1}, \varphi_{2} \in O_{\left(L^{X}, \tau\right)}$. If $x$ is a point in the characterized fuzzy space $\left(X, \varphi_{1,2}\right.$.int $), \mathrm{F} \subseteq X$ and $\boldsymbol{M}$

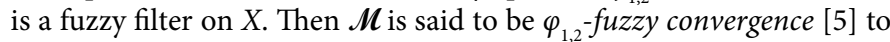
$x$ and written $\boldsymbol{M} \underset{\varphi_{1,2} \text {.int }}{\longrightarrow} x$, provided $\boldsymbol{M}$ is finer than the $\varphi_{1,2}$-fuzzy neighborhood filter $\mathcal{N}_{\varphi_{1,2}}(x)$. Moreover, $\boldsymbol{M}$ is said to be $\varphi_{1,2}$-fuzzy convergence to $\mathrm{F}$ and written $\boldsymbol{M} \underset{\varphi_{1,2} \text {.int }}{\longrightarrow} \mathrm{F}$, provided $\boldsymbol{\mathcal { M }}$ is finer than the $\varphi_{1,2}$-fuzzy neighborhood filter $\mathcal{N}_{\varphi_{1,2}}(x)$ for all $x \in \mathrm{F}$, that is, $\boldsymbol{M}$ is finer than the $\varphi_{1,2}$-fuzzy neighborhood filter $\mathcal{N}_{\varphi_{1,2}}(\mathrm{~F})$.

Internal $\varphi_{1,2}$-closure of fuzzy sets and $\varphi_{1,2}$-closure operators

Let a fuzzy topological space $(X, \tau)$ be fixed and $\varphi_{1}, \varphi_{2} \in O_{\left(L^{X}, \tau\right)}$. The internal $\varphi_{1,2}$-closure of the fuzzy set $\mu: X \rightarrow L$ is the mapping $\varphi_{1,2}, c \mathrm{cl} \mu: X$ $\rightarrow L$ defined by:

$$
\begin{aligned}
& \left(\varphi_{1,2} \cdot \operatorname{cl} \mu\right)(x)=\bigvee_{\mathcal{M} \leq \mathcal{N}_{\varphi_{1}, 2}(x)} \mathcal{M}(\mu), \\
& \left(\varphi_{1,2} \cdot \operatorname{cl} \mu\right)(x)=\bigvee_{\boldsymbol{M} \leq \mathcal{N}_{\varphi_{1,2}}(x)} \boldsymbol{M}(\mu),
\end{aligned}
$$

for all $x \in X$. In (2.6), the fuzzy filter $M$ my have additional properties, e.g, we my assume that is homogeneous or even that is ultra. Obviously, $\varphi_{1,2} \cdot \operatorname{cl} \mu \leq \mu$ holds for all $\mu \in L^{X}$. The mapping $\varphi_{1,2} \cdot \operatorname{cl} F_{L} X \rightarrow F_{L} X$ which assigns $\varphi_{1,2} . \mathrm{cl} M$ to each fuzzy filter $M$ on $X$, that is,

$$
\varphi_{1,2} \cdot \operatorname{cl} \mathcal{M}(\mu)=\underset{\varphi_{1,2} \cdot \operatorname{cl} \rho \leq \mu}{\vee} \boldsymbol{M}(\rho),
$$

is called $\varphi_{1,2}$-closure operator [7] of the characterized fuzzy space $\left(X, \varphi_{1,2}\right.$.int $)$ with respect to the related fuzzy topology $\tau$. Obviously, the $\varphi_{1,2}$-closure operator $\varphi_{1,2}$.cl is isotone hull operator, that is, for all $\boldsymbol{M}, \mathcal{N}$ $\in \mathscr{F}_{L} X$ we have $\boldsymbol{M} \leq \mathcal{N}$ implies $\varphi_{1,2} \cdot \operatorname{cl} \boldsymbol{M} \leq \varphi_{1,2} . \operatorname{cl} N$ and that $\boldsymbol{M} \leq \varphi_{1,2} \cdot \operatorname{cl} \mathcal{M}$.

\section{Lemma 2.2}

Let $(X, \tau)$ be a fuzzy topological space and $\varphi_{1}, \varphi_{2} \in O_{\left(L^{X}, \tau\right)}$. Then for each $x \in X$, we have $\varphi_{1,2} \cdot \operatorname{cl} \dot{x}=\dot{x}$ implies that $\varphi_{1,2} \cdot \operatorname{cl}\{x\}=\{x\}[10]$.

\section{Characterized fuzzy $\boldsymbol{R}_{k}$ and fuzzy $\varphi_{1,2} \boldsymbol{R}_{\boldsymbol{k}}$-spaces}

The notions of characterized fuzzy $R_{k}$ and fuzzy $\varphi_{1,2} R_{k}$-spaces are introduced and studied in Abd-Allah [9,11] for all $k \in\left\{0,1,2 \frac{1}{2}\right\}$. Moreover, the notion of $\varphi_{1,2}$-fuzzy neighborhood filter at the point $x$ and at the ordinary subset of the characterized fuzzy space $\left(X, \varphi_{1,2}\right.$.int $)$ is applied by Abd-Allah [10], to introduced and studied the notions of characterized fuzzy $R_{k}$-spaces for $k \in\{2,3\}$. However, the notions of fuzzy ${ }_{1,2} R_{k}$-spaces are also given by means of the $\varphi_{1,2}$-fuzzy convergence at the point $x$ and at the ordinary subset in the space. We will denote by characterized $F R_{k}$ and $F \varphi_{1,2} R_{k}$-spaces to the characterized fuzzy $R_{k}$ and fuzzy $\varphi_{1,2} R_{k}$-spaces for shorts, respectively.

Let a fuzzy topological space $(X, \tau)$ be fixed and $\varphi_{1}, \varphi_{2} \in O_{\left(L^{X}, \tau\right)}$. Then the characterized fuzzy space $\left(X, \varphi_{1,2}\right.$.int) is said to be:

(1) Characterized $F R_{2}$-space (resp. Characterized $F R_{3}$-space), if for 
Citation: Abd-Allah AS, Al-Khedhairi A (2017) The Relations between Characterized Fuzzy Proximity, Fuzzy Compact, Fuzzy Uniform Spaces and Characterized Fuzzy $T_{s}$-Spaces and Fuzzy $R_{k}$-Spaces. J Appl Computat Math 6: 337. doi: 10.4172/2168-9679.1000337

Page 6 of 15

all $x \in X, \mathrm{~F} \varphi_{1,2} C(X)$ such that $x \ddot{\mathrm{I}} \mathrm{F}$ (resp. $\mathrm{F}_{1}, \mathrm{~F}_{2} \in \varphi_{1,2} C(X)$ such that $\left.\mathrm{F}_{1} \cap \mathrm{F}_{2}=\emptyset\right)$, the infimum $\mathcal{N}_{\varphi_{1,2}}(x) \wedge \mathcal{N}_{\varphi_{1,2}}(\mathrm{~F})\left(\right.$ resp. $\left.\mathcal{N}_{\varphi_{1,2}}\left(\mathrm{~F}_{1}\right) \wedge \mathcal{N}_{\varphi_{1,2}}\left(\mathrm{~F}_{2}\right)\right)$ does not exists. The related fuzzy topological space $(X, \tau)$ is said to be $\mathrm{F} \varphi_{1,2} R_{2}$-space (resp. $\mathrm{F} \varphi_{1,2} R_{3}$-space) if for all $x \in X$ (resp. $\mathrm{F} \in \varphi_{1,2} \cdot C(X)$ ) and $\boldsymbol{M} \in \mathscr{F}_{L} X$ such that $\boldsymbol{M} \underset{\varphi_{1,2} \text {.int }}{\longrightarrow} x \quad$ (resp. $\mathcal{M} \underset{\varphi_{1,2} \text {.int }}{\longrightarrow} \mathrm{F}$ ) we have $\varphi_{1,2} . \operatorname{cl} \mathcal{M} \underset{\varphi_{1,2} \text {.int }}{\longrightarrow} x$ (resp. $\varphi_{1,2} \cdot$ cl $\left.\mathcal{M} \underset{\varphi_{1,2} \text {.int }}{\longrightarrow} \mathrm{F}\right)$.

(2) Characterized $F R_{2 \frac{1}{2}}$ - space if for all $x \in X, \mathrm{~F} \in \varphi_{1,2} C(X)$ such that $x$ Ï F, there exists an $\varphi_{1,2} \psi_{1,2}$-fuzzy continuous mapping $f:\left(X, \varphi_{1,2}\right.$.int $) \rightarrow$ $\left(I_{L}, \psi_{1,2}\right.$.int $)$ such that $f(x)=\overline{1}$ and $f(y)=\overline{0}$ for all $y \in \mathrm{F}$. The related fuzzy topological space $(X, \tau)$ is said to be $F \varphi_{1,2} R_{2 \frac{1}{2}}$ - space if and only if $\left(X, \varphi_{1,2}\right.$.int $)$ is characterized $F R_{2 \frac{1}{2}}$ - space.

\section{Characterized fuzzy $T_{s}$ and fuzzy $\varphi_{1,2}-T_{S}$ spaces}

The notions of characterized fuzzy $T_{s}$ and fuzzy $\varphi_{1,2}-T_{S}$ spaces are investigated and studied by Abd-Allah and by Abd-Allah and Al-Khedhairi in $[8,9,11]$ for all $s \in\left\{0,1,2,2 \frac{1}{2}, 3,3 \frac{1}{2}, 4\right\}$. These characterized fuzzy spaces depend only on the usual points and the operation defined on the class of all fuzzy subsets of $X$ endowed with a fuzzy topological space $(X, \tau)$. We will denote by characterized $F T_{s}$ and $F \varphi_{1,2}-T_{s}$ spaces to the characterized fuzzy $T_{s}$ and fuzzy $\varphi_{1,2}-T_{S}$ spaces for shorts, respectively.

Let a fuzzy topological space $(X, \tau)$ be fixed and $\varphi_{1}, \varphi_{2} \in O_{\left(L^{X}, \tau\right)}$. Then the characterized fuzzy space $\left(X, \varphi_{1,2}\right.$.int $)$ is said to be:

(1) Characterized $F T_{0}$-space (resp. Characterized $F T_{1}$-space) if for all $x, y \in X$ such that $x \neq y$ there exist $\mu \in L^{X}$ and $\alpha \in L_{0}$ such that $\mu(x)<$ $\alpha \leq\left(X, \varphi_{1,2}\right.$.int $\left.\mu\right)(y)$ holds or (resp. and) there exist $\rho \in L^{X}$ and $\beta \in L_{0}$ such that $\rho(y)<\beta \leq\left(\varphi_{1,2}\right.$.int $\left.\rho\right)(x)$ holds. The related fuzzy topological space $(X, \tau)$ is said to be $F \varphi_{1,2}-T_{0}$ space (resp. $F \varphi_{1,2}-T_{1}$ space) if for all $x, y \in X$ such that $x \neq y$ we have $\dot{x} \nless \mathcal{N}_{\varphi_{1,2}}(y)$ or (resp. and) $\dot{y} \nless \leq \mathcal{N}_{\varphi_{1,2}}(x)$.

(2) Characterized $F T_{2}$-space if for all $x, y \in X$ such that $x \neq y$, the infimum $\mathcal{N}_{\varphi_{1,2}}(x) \wedge \mathcal{N}_{\varphi_{1,2}}(y)$ does not exists. The related fuzzy topological space $(X, \tau)$ is said to be $F \varphi_{1,2}-T_{2}$ space if $\mathcal{M} \underset{\varphi_{1,2} \text { int }}{\longrightarrow} x, y$ implies $x=y$ for all $\boldsymbol{M} \in \mathscr{F}_{L} X$ and for all $x, y \in X$.

(3) Characterized $F T_{s}$ space if and only if it is characterized $F R_{k}$ space and characterized $F T_{1}$-space for $k \in\left\{2,2 \frac{1}{2}, 3\right\}$ and $s \in\left\{3,3 \frac{1}{2}, 4\right\}$. The related fuzzy topological space $(X, \tau)$ is said to be $F \varphi_{1,2}-T_{s}$ if and only if it is $F \varphi_{1,2}-R_{k}$ and $F \varphi_{1,2}-T_{1}$.

\section{Proposition 2.4}

Let $(X, \tau)$ be an fuzzy topological space and $\varphi_{1}, \varphi_{2} \in O_{\left(L^{X}, \tau\right)}$. Then the characterized fuzzy space $\left(X, \varphi_{1,2}\right.$.int) is characterized $F T_{1}$-space if and only if $\varphi_{1,2} . \mathrm{cl} \dot{x}=\dot{x}$ for all $x \in X[8]$.

\section{Proposition 2.5}

If $\left(X, \varphi_{1,2}\right.$.int $)$ is characterized $F T_{2}$-space and $\varphi_{1,2}$ int is finer than $\psi_{1,2}$. int, then $\left(X, \psi_{1,2}\right.$ int $)$ is also characterized $F T_{2}$-space [8].

\section{Proposition 2.6}

Let a fuzzy topological space $(X, \tau)$ be fixed and $\varphi_{1}, \varphi_{2} \in O_{\left(L^{X}, \tau\right)}$. Then the following are fulfilled $[8,22]$ :
(1) Every characterized $F T_{i}$-space $\left(X, \varphi_{1,2}\right.$ int) is characterized $F T_{i-1}{ }^{-}$ space for each $i \in\{2,3,4\}$.

(2) The characterized fuzzy subspace and the characterized fuzzy product space of a family of characterized $F T_{2}$-spaces are also characterized $\mathrm{FT}_{2}$-spaces .

\section{New Relations between Characterized $F T_{s}$, Characterized $F R_{k}$ and Characterized Fuzzy Proximity Spaces}

In this section we are going to introduce and study the relations between the characterized $F T_{s}$-spaces, the characterized $F R_{k}$-spaces and the characterized fuzzy proximity spaces presented by Abd-Allah in [12]. We make at first the relation between the farness on fuzzy sets and the finer relation on fuzzy filters. So, we show some results for the notion of the $\varphi_{1,2}$-fuzzy neighborhood filter $\mathcal{N}_{\varphi_{1,2}}(\mu)$ at the fuzzy subset $\mu \in L^{X}$. The notion of homogeneous fuzzy filter $\dot{\mu}$ which is defined in (2.1) and the notion of $\varphi_{1,2}$-fuzzy neighborhood filter $\mathcal{N}_{\varphi_{1,2}}(\mu)$ at the fuzzy subset $\mu \in L^{X}$ are applied at first to study the relation between the fuzzy proximity $\delta$ defined by Katsaras in [18] and our fuzzy separation axioms [8-10]. Moreover, the relations between characterized fuzzy proximity spaces and the characterized $F T_{s}$-spaces and characterized $F R_{k}$-spaces are introduced for $s \in\left\{0,1,2,3,3 \frac{1}{2}, 4\right\}$ and $k \in\left\{1,2,2 \frac{1}{2}, 3\right\}$.

\section{Proposition 3.1}

Let a fuzzy topological space $(X, \tau)$ be fixed and $\varphi_{1}, \varphi_{2} \in O_{\left(L^{X}, \tau\right)}$ such that $\varphi_{2} \geq 1_{L^{X}}$ is isotone and idempotent and $\varphi_{1}$ is wfip with respect to $\varphi_{1} \mathrm{OF}(X)$. Then the supremum of the $\varphi_{1,2}$-fuzzy neighborhood filters $\mathcal{N}_{\varphi_{1,2}}(x)$ at $x \in X$ which is given by:

$$
\mathcal{N}_{\varphi_{1,2}}(\mu)=\bigvee_{0<\mu(x)} \mathcal{N}_{\varphi_{1,2}}(x),
$$

for all $\mu \in L^{X}$ is a fuzzy filter on $X$ called a $\varphi_{1,2}$-fuzzy neighborhood filter at $\mu$.

Proof: Fix an $\alpha \in L_{0}$, then because of (2.4) and the condition $\varphi_{2} \geq 1_{L^{X}}$, we have

$$
\begin{aligned}
& \mathcal{N}_{\varphi_{1,2}}(\bar{\alpha})=\bigwedge_{0<\mu(y)} \mathcal{N}_{\varphi_{1,2}}(y)(\bar{\alpha})=\bigwedge_{0<\mu(y)}\left(\varphi_{1,2} \cdot \operatorname{int} \bar{\alpha}\right)(y) \leq \bigwedge_{0<\mu(y)} \bar{\alpha}(y)=\alpha \\
& \text { and } \\
& \mathscr{N}_{\varphi_{1,2}}(\overline{1})=\bigwedge_{0<\mu(y)}\left(\varphi_{1,2} \cdot \operatorname{int} \overline{1}\right)(y)=\bigwedge_{0<\mu(y)} \overline{1}(y)=1 .
\end{aligned}
$$

Thus, condition $\left(F_{1}\right)$ is fulfilled. To prove condition $\left(F_{2}\right)$, let $\rho, \eta \in L^{X}$, then because of Proposition 2.4 and (2.4) we have

$$
\begin{aligned}
\mathcal{N}_{\varphi_{1,2}}(\mu)(\rho \wedge \eta) & =\bigwedge_{0<\mu(y)} \varphi_{1,2} \cdot \operatorname{int}(\rho \wedge \eta)(y) \\
& =\bigwedge_{0<\mu(y)}\left(\varphi_{1,2} \cdot \operatorname{int} \rho\right)(y) \wedge \bigwedge_{0<\mu(y)}\left(\varphi_{1,2} \cdot \operatorname{int} \eta\right)(y) \\
& =\mathcal{N}_{\varphi_{1,2}}(\mu)(\rho) \wedge \mathcal{N}_{\varphi_{1,2}}(\mu)(\eta) .
\end{aligned}
$$

Hence, $\mathcal{N}_{\varphi_{1,2}}(\mu)$ is a fuzzy filter on $X$. Since $\left(\varphi_{1,2}\right.$.int $)(x) \leq \rho(x)$ holds for all $x \in X$ and $\rho \in L^{X}$, then $\mathcal{N}_{\varphi_{1,2}}(\mu)(\rho) \leq \dot{\mu}(\rho)$ holds for all $\rho \in \in L^{X}$. Thus, $\dot{\mu} \leq \mathcal{N}_{\varphi_{1,2}}(\mu)$ and therefore $\mathcal{N}_{\varphi_{1,2}}(\mu)$ fulfills condition $\left(\mathrm{N}_{1}\right)$. For condition $\left(\mathrm{N}_{2}\right)$, let $\rho, \eta \in L^{X}$ such that $\rho \leq \eta$. Because of Proposition 2.4, we have $\varphi_{1,2}$.int $\rho \leq \varphi_{1,2}$.int $\eta$ holds and which implies that $\bigwedge_{0<\mu(y)}\left(\varphi_{1,2}\right.$.int $\left.\rho\right)(y) \leq \bigwedge_{0<\mu(y)}\left(\varphi_{1,2}\right.$.int $\left.\eta\right)(y)$ holds for all $y \in X$. Hence $\mathcal{N}_{\varphi_{1,2}}(\mu)(\rho) \leq \mathcal{N}_{\varphi_{1,2}}(\mu)(\eta)$ and therefore condition $\left(\mathrm{N}_{2}\right)$ is fulfilled. Since for any $y \in X$ we have $\bigwedge_{0<\mu(y)} y \mapsto \bigwedge_{0<\mu(y)}\left(\varphi_{1,2}\right.$.int $\left.\rho\right)(y)$ represents 
Citation: Abd-Allah AS, Al-Khedhairi A (2017) The Relations between Characterized Fuzzy Proximity, Fuzzy Compact, Fuzzy Uniform Spaces and Characterized Fuzzy $T_{s}$-Spaces and Fuzzy $R_{k}$-Spaces. J Appl Computat Math 6: 337. doi: 10.4172/2168-9679.1000337

Page 7 of 15

the mapping $\varphi_{1,2}$ int $\rho$. Then from Proposition 2.4 we have

$$
\mathcal{N}_{\varphi_{1,2}}(\mu)\left(\varphi_{1,2} \cdot \operatorname{int} \rho\right)=\bigwedge_{0<\mu(x)} \varphi_{1,2} \cdot \operatorname{int}\left(\varphi_{1,2} \cdot \operatorname{int} \rho\right)(x)=\bigwedge_{0<\mu(x)}\left(\varphi_{1,2} \cdot \operatorname{int} \rho\right)(x),
$$

and then $\mathscr{N}_{\varphi_{1,2}}(\mu)\left(\bigwedge_{0<\mu(y)} y \mapsto \bigwedge_{0<\mu(y)} \varphi_{1,2}\right.$.int $\left.\rho(y)\right)=\mathcal{N}_{\varphi_{1,2}}(\mu)(\rho)$ for all $y \in X$ and $\rho \in L^{X}$. Thus, condition (N3) is also fulfilled and therefore $\mathcal{N}_{\varphi_{1,2}}(\mu)$ fulfilled the conditions $\left(\mathrm{N}_{1}\right)$ to $\left(\mathrm{N}_{3}\right)$ of the $\varphi_{1,2}$-fuzzy neighborhood filters.

Not that in Bayoumi et al. [15], the supremum of the empty set of the fuzzy filters is the finest fuzzy filter. This means $\mathcal{N}_{\varphi_{12}}(\overline{0}) \leq \dot{\mu}$ for all $\mu \in L^{X}$. Because of (2.4) the equations (2.1) and (2.2) can be written as in the following:

$$
\begin{aligned}
& \dot{\mu}(\rho)=\bigwedge_{0<\mu(x)} \rho(x), \\
& \left.\mathcal{N}_{\varphi_{1,2}}(\mu)(\rho)=\bigwedge_{0<\mu(x)} \mathcal{N}_{\varphi_{1,2}}(x)(\rho)=\widehat{0<\mu(x)}_{\left(\varphi_{1,2}\right.} \cdot \operatorname{int} \rho\right)(x),
\end{aligned}
$$

for all $\rho \in L^{X}$. Here a useful remark is given

Remark 3.1: The homogeneous fuzzy filter $\dot{x}$ at the ordinary point $x$ is nothing that a homogeneous fuzzy filter $\dot{\mathrm{X}}$ at the fuzzy point $x_{\alpha}$, that is, $\dot{x}_{\alpha}=\dot{\mathrm{x}}$ for all $x \in X$ and $\alpha \in L_{0}$. Moreover, the $\varphi_{1,2}$-fuzzy neighborhood filter $\mathcal{N}_{\varphi_{1,2}}(x)$ at $x$ is itself the $\varphi_{1,2}$-fuzzy neighborhood filter $\mathcal{N}_{\varphi_{1,2}}\left(x_{\alpha}\right)$ at $x_{\alpha}$

The $\varphi_{1,2}$-fuzzy neighborhood filter $\mathscr{N}_{\varphi_{1,2}}(\mu)$ at the fuzzy subset $\mu \in L^{X}$ and the homogeneous fuzzy filter ${ }^{\mu} \dot{\mu}$ fulfill the following properties.

\section{Lemma 3.1}

Let $(X, \tau)$ be a fuzzy topological space and $\varphi_{1}, \varphi_{2} \in O_{\left(L^{X}, \tau\right)}$. Then for all $\mu, \rho \in L^{X}$ the following properties are fulfilled:

(1) $\dot{\mu} \leq \dot{\rho}$ implies $\mathscr{N}_{\varphi_{1,2}}\left(\rho^{\prime}\right) \leq \dot{\mu}^{\prime}$ and $\mathscr{N}_{\varphi_{1,2}}(\mu) \leq \dot{\rho}$ implies $\mathcal{N}_{\varphi_{1,2}}\left(\rho^{\prime}\right) \leq \dot{\mu}^{\prime}$.

(2) $\mu \leq$ implies $\mathscr{N}_{\varphi_{1,2}}(\mu) \leq \mathcal{N}_{\varphi_{1,2}}(\rho)$.

(3) $\mathcal{N}_{\varphi_{1,2}}(\mu \vee \rho)=\mathcal{N}_{\varphi_{1,2}}(\mu) \wedge \mathcal{N}_{\varphi_{1,2}}(\rho)$.

(4) $\mathcal{N}_{\varphi_{1,2}}(\mu) \leq \dot{\rho}$ implies $\mu \leq \rho$.

(5) $\mathcal{N}_{\varphi_{1,2}}(\mu) \leq \dot{\rho}$ implies there is an $\eta \in L^{X}$ such that $\mathcal{N}_{\varphi_{1,2}}(\mu) \leq \dot{\eta}$ and $\mathcal{N}_{\varphi_{1,2}}(\eta) \leq \dot{\rho}$.

Proof: Let $\dot{\mu} \leq \dot{\rho}$. From condition(N1) wehave $\dot{\mu} \leq \mathcal{N}_{\varphi_{1,2}}(\rho)$ holds and therefore for all $\eta \in L^{X}$ we have $\widehat{\Upsilon}_{0<\mu(x)} \eta(x) \geq \widehat{\bigwedge}_{0<\rho(y)}\left(\varphi_{1,2} \cdot \operatorname{int} \eta\right)(y)$ holds. Hence, ${\widehat{0<\mu^{\prime}(x)}} \eta(x) \leq \widehat{\Upsilon}_{0<\rho^{\prime}(y)}\left(\varphi_{1,2} \cdot \operatorname{int} \eta\right)(y)$ holds also. Thus, $\dot{\mu}^{\prime}(\eta) \leq \mathcal{N}_{\varphi_{1,2}}\left(\rho^{\prime}\right)(\eta)$ and $\mathcal{N}_{\varphi_{1,2}}\left(\rho^{\prime}\right) \leq \dot{\mu}^{\prime}$ are hold. Similarly, if $\mathscr{N}_{\varphi_{1,2}}(\mu) \leq \dot{\rho}$, then from (N1) we have $\dot{\mu} \leq \dot{\rho}$ which implies $\mathcal{N}_{\varphi_{1,2}}\left(\rho^{\prime}\right) \leq \dot{\mu}^{\prime}$. Thus, (1) is fulfilled. Since $\mu \leq \rho$ implies $\mu(x) \leq \rho(x)$ for all $x X$, then

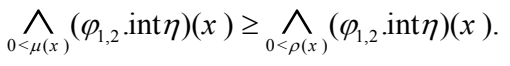

Hence, $\mathcal{N}_{\varphi_{1,2}}(\mu)(\eta) \geq \mathcal{N}_{\varphi_{1,2}}(\rho)(\eta)$ holds for all $\eta \in L^{X}$ and therefore $\mathcal{N}_{\varphi_{1,2}}(\mu) \leq \mathcal{N}_{\varphi_{1,2}}(\rho)$ holds. Hence, (2) is fulfilled.

Since $\mu, \rho \leq \mu$ U $\rho$, then from (2) we have
$\mathcal{N}_{\varphi_{1,2}}(\mu) \wedge \mathcal{N}_{\varphi_{1,2}}(\rho) \leq \mathcal{N}_{\varphi_{1,2}}(\mu \vee \rho)$. Now, let $\eta \in L^{X}$ then

$$
\begin{aligned}
& \left(\mathcal{N}_{\varphi_{1,2}}(\mu) \wedge \mathcal{N}_{\varphi_{1,2}}(\rho)\right)(\eta)=\underset{k_{1} \wedge k_{2} \leq \eta}{\bigvee}\left(\mathcal{N}_{\varphi_{1,2}}(\mu)\left(k_{1}\right) \wedge \mathcal{N}_{\varphi_{1,2}}(\rho)\left(k_{2}\right)\right) \\
& =\underset{k_{1} \wedge k_{2} \leq \eta}{\bigvee}\left(\bigwedge_{0<\mu(x)} \varphi_{1,2} \cdot \operatorname{int} k_{1}(x) \wedge \bigwedge_{0<\rho(y)} \varphi_{1,2} \cdot \operatorname{int} k_{2}(y)\right) \\
& \leq \underset{k_{1} \wedge k_{2} \leq \eta}{\vee} \bigwedge_{0<(\mu \vee \rho)(z)} \varphi_{1,2} \cdot \operatorname{int}\left(k_{1} \wedge k_{2}\right)(z) \\
& \leq \bigwedge_{0<(\mu \vee \rho)(z)} \varphi_{1,2} \cdot \operatorname{int} \eta(z)=\mathcal{N}_{\varphi_{1,2}}(\mu \vee \rho)(\eta) .
\end{aligned}
$$

Hence, $\mathcal{N}_{\varphi_{1,2}}(\mu) \wedge \mathcal{N}_{\varphi_{1,2}}(\rho) \geq \mathscr{N}_{\varphi_{1,2}}(\mu \vee \rho)$ holds and therefore (3) is fulfilled. To prove (4), let $\mathcal{N}_{\varphi_{1,2}}(\mu) \leq \dot{\rho}$ holds. Because of (2.1), (3.1) and (N1), we have $\dot{\mu} \leq \mathscr{N}_{\varphi_{1,2}}(\mu)$ and then $\dot{\mu} \leq \dot{\rho}$ holds. Hence, Proposition 2.1 implies $\mu \leq \rho$. Thus, (4) is fulfilled. Finally,

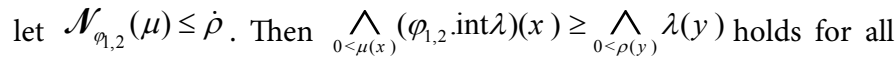
$\lambda \in L^{X}$. Hence, there is $\eta \in L^{X}$ such that

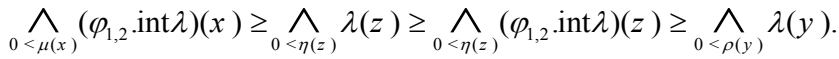

This means there is $\eta \in L^{X}$ such that $\mathscr{N}_{\varphi_{1,2}}(\mu)(\lambda) \geq \dot{\eta}(\lambda)$ and $\mathcal{N}_{\varphi_{1,2}}(\eta)(\lambda) \geq \dot{\rho}(\lambda)$ are hold for all $\lambda \in L^{X}$. Thus, $\mathcal{N}_{\varphi_{1,2}}(\mu) \leq \dot{\eta}$ and $\mathcal{N}_{\varphi_{1,2}}(\eta) \leq \dot{\rho}$ are also hold. Consequently, (5) is fulfilled.

In the characterized fuzzy space $\left(X, \varphi_{1,2}\right.$.int), the fuzzy proximity will be identified with the finer relation on the fuzzy filters, specially with the finer relation on the $\varphi_{1,2}$-fuzzy neighborhood filters. This shown in the following proposition.

\section{Proposition 3.2}

Let $(X, \tau)$ be a fuzzy topological space and $\varphi_{1}, \varphi_{2} \in O_{\left(L^{X}, \tau\right)}$. Then the binary relation $\delta$ on $L^{X}$ which is defined by:

$\mu \bar{\delta} \rho$ if and only if $\mathscr{N}_{\varphi_{1,2}}(\rho) \leq \dot{\mu}^{\prime}$,

for all $\mu, \rho \in L^{X}$ is fuzzy proximity on $X$.

Proof: Let $\mu, \rho \in L^{X}$ such that $\mu \bar{\delta} \rho$, then $\mathscr{N}_{\varphi_{1,2}}(\rho) \leq \dot{\mu}^{\prime}$. Because of (1) in Lemma 3.1, we have $\mathcal{N}_{\varphi_{1,2}}(\mu) \leq \dot{\rho}^{\prime}$ and therefore $\rho \bar{\delta} \mu$. Hence, condition (P1) is fulfilled.

Since $\mathcal{N}_{\varphi_{1,2}}(\mu) \leq \mathcal{N}_{\varphi_{1,2}}(\mu \vee \rho)$ and $\mathcal{N}_{\varphi_{1,2}}(\rho) \leq \mathcal{N}_{\varphi_{1,2}}(\mu \vee \rho)$ are hold for all $\mu, \rho \in L^{X}$, then $\mathcal{N}_{\varphi_{1,2}}(\mu \vee \rho) \leq \dot{\eta}^{\prime}$ implies $\mathcal{N}_{\varphi_{1,2}}(\mu) \leq \dot{\eta}^{\prime}$ and $\mathcal{N}_{\varphi_{1,2}}(\rho) \leq \dot{\eta}^{\prime}$ are hold for all $\eta \in L^{X}$. This means $\eta \bar{\delta}(\mu \vee \rho)$ implies $\eta \bar{\delta} \mu$ and $\eta \bar{\delta} \rho$. Conversely, let $\eta \bar{\delta} \mu$ and $\eta \bar{\delta} \rho$ for all $\mu, \rho, \eta \in L^{X}$, then $\mathscr{N}_{\varphi_{1,2}}(\mu) \leq \dot{\eta}^{\prime}$ and $\mathcal{N}_{\varphi_{1,2}}(\rho) \leq \dot{\eta}^{\prime}$ are hold. Hence, (3) in Lemma 3.1 implies $\mathscr{N}_{\varphi_{1,2}}(\mu \vee \rho)=\mathscr{N}_{\varphi_{1,2}}(\mu) \wedge \mathcal{N}_{\varphi_{1,2}}(\rho) \leq \dot{\eta}^{\prime} \quad$ holds and therefore $\eta \bar{\delta}(\mu \vee \rho)$. Consequently, (P2) is fulfilled. To prove (P3), since $\mathscr{N}_{\varphi_{1,2}}(\overline{0}) \leq \dot{\mu}^{\prime}$ holds for all $\mu \in \in L^{X}$. Then, $\mu \bar{\delta} \overline{0}$ for all $\mu \in L^{X}$. Hence, $\mu=\overline{0}$ or $\rho=\overline{0}$ implies $\mu \bar{\delta} \rho$ for all $\rho, \eta \in L^{X}$. Thus, (P3) is fulfilled.

Let $\mu, \rho \in L^{X}$ such that $\mu \bar{\delta} \rho$, then $\mathcal{N}_{\varphi_{1,2}}(\rho) \leq \dot{\mu}^{\prime}$. Because of (1) and (4) in Lemma 3.1, we have $\mathcal{N}_{\varphi_{1,2}}(\mu) \leq \dot{\rho}^{\prime}$ and therefore $\mu \leq$ $\rho^{\prime}$, that is, (P4) is fulfilled. Finally, let $\mu, \rho \in L^{X}$ such that $\mu \bar{\delta} \rho$, then 
Citation: Abd-Allah AS, Al-Khedhairi A (2017) The Relations between Characterized Fuzzy Proximity, Fuzzy Compact, Fuzzy Uniform Spaces and Characterized Fuzzy $T_{s}$-Spaces and Fuzzy $R_{k}$-Spaces. J Appl Computat Math 6: 337. doi: 10.4172/2168-9679.1000337

$\mathcal{N}_{\varphi_{1,2}}(\rho) \leq \dot{\mu}^{\prime}$ which implies $\mathcal{N}_{\varphi_{1,2}}(\mu) \leq \dot{\rho}^{\prime}$. Because of (5) in Lemma 3.1, there is an $\eta \in L^{X}$ such that $\mathscr{N}_{\varphi_{1,2}}(\mu) \leq \dot{\eta}$ and $\mathscr{N}_{\varphi_{1,2}}(\eta) \leq \dot{\rho}^{\prime}$ are hold. Hence, $\mathcal{N}_{\varphi_{1,2}}\left(\eta^{\prime}\right) \leq \dot{\mu}^{\prime}$ and $\mathcal{N}_{\varphi_{1,2}}(\rho) \leq \dot{\eta}^{\prime}$ are also hold, that is, $\mu \bar{\delta} \eta^{\prime}$ and $\eta \bar{\delta} \rho$. Thus, (P5) holds and consequently, $\delta$ is fuzzy proximity on $X$.

If a fuzzy topological space $(X, \tau)$ be fixed and $\varphi_{1}, \varphi_{2} \in O_{\left(L^{X}, \tau\right)}$. Then each fuzzy proximity $\delta$ on $X$ is associated a set of all $\varphi_{1,2}$-open fuzzy subsets of $X$ with respect to $\delta$ denoted by $\varphi_{12} O F(X)_{\delta}$. In this case the triple $\left(X, \varphi_{1,2} O F(X)_{\delta}\right)$ as will as $\left(X, \varphi_{1,2}\right.$. int $\left._{\delta}\right)$ is said to be characterized fuzzy proximity space. The related $\varphi_{1,2}$-interior and $\varphi_{1,2}$-closure operators $\varphi_{1,2}$.int ${ }_{\delta}$ and $\varphi_{1,2} \cdot \mathrm{cl}_{\delta}$ are given by:

$$
\varphi_{1,2} \cdot \text {.nt }_{\delta} \mu=\bigvee_{\mu^{\prime} \delta} \rho \text { and } \varphi_{1,2} \cdot \mathrm{cl}_{\delta} \mu=\bigwedge_{\mu^{\prime} \delta} \rho
$$

respectively, for all $\mu \in L^{X}$. Consider the characterized fuzzy proximity space $\left(X, \varphi_{1,2}\right.$. int $\left._{\delta}\right)$ be fixed and $\mu \in L^{X}$, then $\mu$ is said to be $\varphi_{1,2} \delta$-fuzzy neighborhood for the point $x \in X$ if and only if $x_{1} \bar{\delta} \mu^{\prime}$. Moreover, the mapping $f:\left(X, \varphi_{1,2} \cdot\right.$.nt $\left._{\delta}\right) \rightarrow\left(Y, \psi_{1,2} \cdot\right.$.int $\left._{\delta^{*}}\right)$ is said to be $\varphi_{1,2} \psi_{1,2} \delta$-fuzzy continuous, provided $\eta \bar{\delta}^{*} \rho$ implies $(\eta \circ f) \bar{\delta}(\rho \circ f)$ for all $\eta, \rho \in L^{Y}$.

In the following we will show that the characterized fuzzy proximity space $\left(X, \varphi_{1,2}\right.$.int $\left.{ }_{\delta}\right)$ is characterized $F T_{0}$-space as in sense of [8] if and only if $\delta$ is separated.

\section{Proposition 3.3}

Let $(X, \tau)$ be a fuzzy topological space, $\varphi_{1} \varphi_{2} \in$, , and is a fuzzy proximity on $X$. Then the characterized fuzzy proximity space $\left(X, \varphi_{12}\right.$. int $_{\delta}$ ) is characterized $F T_{0}$-space if and only if $\delta$ is separated.

Proof: Let $\left(X, \varphi_{1,2} \cdot\right.$ int $\left._{\delta}\right)$ is characterized $F T_{0}$-space and let $x, y \in X$ such that $x \neq y$. Then $\dot{x} \not \mathscr{N}_{\varphi_{1,2}}^{\delta}(y)$ and therefore there is $\mu \in L^{X}$ such that $\varphi_{1,2}$.int ${ }_{\delta} \mu(y)>\mu(x)$. Because of (3.4), we have $\underset{\mu^{\prime} \delta}{\bigvee} \rho(y)>\mu(x)$ and hence $\mu(x)>\rho(y)$ holds for all $\rho L^{X}$ with $\mu^{\prime} \bar{\delta} \rho$, that is, $\mu(x)>\rho$ $(y)$ holds for all $\rho \in \in L^{X}$ with $\mathscr{N}_{\varphi_{1,2}}^{\delta}(\rho) \leq \dot{\mu}$. Choose $\mu=x_{1}^{\prime}$ and ${ }^{\mathscr{W}_{\alpha 2}^{s}\left(y_{1}\right) \leq x_{1}^{\prime}}$, then because of Remark 3.1, we get $\mathscr{N}_{\varphi_{1,2}}^{\delta}\left(y_{1}\right) \leq x_{1}^{\prime}$. Using Proposition 3.2 we get $x_{1} \bar{\delta} y_{1}$ and therefore $x_{\alpha} \bar{\delta} y_{\beta}$ holds for all $\alpha, \beta \in L_{0}$. Thus, $\delta$ is separated.

Conversely, let $\delta$ is separated fuzzy proximity and let $x, y \in X$ such that $x \neq y$. Then, $x_{1} \bar{\delta} y_{1}$ and because of Proposition 3.2 and Remark 3.1, we have $\mathscr{N}_{\varphi_{1,2}}^{\delta}(y) \leq \dot{x}^{\prime}$. Therefore, $\varphi_{1,2} \cdot$.nt $_{\delta} \mu(y) \geq \bigwedge_{z \neq x} \mu(z)$ holds for all $\mu \in L^{X}$. Consider, $\mu=x_{1}^{\prime}$ we get $\varphi_{1,2} \cdot$ int $_{\delta} x_{1}^{\prime}(y)=1$ and $x_{1}^{\prime}(x)=0$. Hence, there exists $\mu=x_{1}^{\prime} \in L^{X}$ such that $\varphi_{1,2} \cdot$ int $_{\delta} \mu(y)=1>\mu(x)$, that is, $\dot{x} \not \mathscr{N}_{\varphi_{1,2}}^{\delta}(y)$ and therefore $\left(X, \varphi_{1,2}\right.$.int $)$ is characterized $F T_{0}$-space.

In the following proposition, the ${ }_{1,2}$-closure of the fuzzy subsets in the characterized fuzzy space $\left(X, \varphi_{1,2} \cdot\right.$ int $\left._{\delta}\right)$ are equivalent with the fuzzy subsets by the fuzzy proximity $\delta$ on $X$.

\section{Proposition 3.4}

Let $(X, \tau)$ be a fuzzy topological space, $\varphi_{1}, \varphi_{2} \in O_{\left(L^{X}, \tau\right)}$ such that $\varphi_{2} \geq 1_{L^{X}}$ and $\delta$ is a fuzzy proximity on $X$. Then, $\mu \bar{\delta} \rho$ if and only if $\varphi_{1,2} \cdot \mathrm{cl}_{\delta} \mu \bar{\delta} \varphi_{1,2} . \mathrm{cl}_{\delta} \rho$ for all $\mu, \rho \in L^{X}$.

Proof: Let $\mu, \rho \in L^{X}$ such that $\varphi_{1,2} \cdot \mathrm{cl}_{\delta} \mu \bar{\delta} \varphi_{1,2} \cdot \mathrm{cl}_{\delta} \rho$, then Proposition
3.2 implies $\mathcal{N}_{\varphi_{1,2}}\left(\varphi_{1,2} \cdot \mathrm{cl}_{\delta} \rho\right) \leq\left(\varphi_{1,2} \cdot \mathrm{cl}_{\delta} \mu\right)^{\prime}$. Since $\varphi_{2} \geq 1_{L^{X}}$ and $\mathcal{N}_{\varphi_{1,2}}(\eta)$ is isotone operator, then $\mu \leq \varphi_{1,2} \cdot{ } \mathrm{cl}_{\delta} \mu$ and $\mathcal{N}_{\varphi_{1,2}}(\rho) \leq \mathcal{N}_{\varphi_{1,2}}\left(\varphi_{1,2} \cdot \mathrm{cl}_{\delta} \rho\right)$ are hold for all $\mu, \rho \in L^{X}$. Hence, $\mathcal{N}_{\varphi_{1,2}}(\rho) \leq \dot{\mu}^{\prime}$ and therefore $\mu \bar{\delta} \rho$.

Conversely, Let $\mu, \rho \in L^{X}$ such that $\mu \bar{\delta} \rho$. Because of Proposition 3.2 we have $(\rho) \leq \mu^{\prime}$. Since $\varphi_{2} \geq 1_{L^{X}}$ and $\mathcal{N}_{\varphi, 2}^{\delta}(\eta)$ is isotone operator, then $\mu^{\prime} \leq \varphi_{1,2} \cdot \mathrm{cl}_{\delta} \mu^{\prime}$ holds for all $\mu^{\prime} \in L^{X}$ and therefore $\dot{\rho} \leq \mathcal{N}_{\varphi_{1,2}}^{\delta}\left(\mu^{\prime}\right) \leq \mathcal{N}_{\varphi_{1,2}}^{\delta}\left(\varphi_{1,2} \cdot \mathrm{cl}_{\delta} \mu^{\prime}\right)$. From Lemma 3.1, we have $\mathcal{N}_{\varphi_{1,2}}^{\delta}\left(\varphi_{1,2} \cdot \mathrm{cl}_{\delta} \mu\right) \leq \dot{\rho}^{\prime}$ and then $\rho \bar{\delta} \varphi_{1,2} \cdot \mathrm{cl}_{\delta} \mu$ . Therefore, $\varphi_{1,2} \cdot \mathrm{cl}_{\delta} \mu \leq \mathcal{N}_{\varphi_{1,2}}^{\delta}\left(\rho^{\prime}\right)$ holds. Using Lemma 3.1 we get $\varphi_{1,2} \cdot \mathrm{cl}_{\delta} \mu \leq \mathcal{N}_{\varphi, 2}^{\delta}\left(\rho^{\prime}\right) \leq \mathcal{N}_{\varphi, 2}^{\delta}\left(\varphi_{1,2} \cdot \mathrm{cl}_{\delta} \rho^{\prime}\right)$. Thus, $\mathcal{N}_{\varphi, 2}^{\delta}\left(\varphi_{1,2} \cdot \mathrm{cl}_{\delta} \rho\right) \leq\left(\varphi_{1,2} \cdot \mathrm{cl}_{\delta} \mu\right)^{\prime}$ and therefore $\varphi_{1,2} \cdot \mathrm{cl}_{\delta} \mu \bar{\delta} \varphi_{1,2} \cdot \mathrm{cl}_{\delta} \rho$ for all $\mu, \rho \in L^{X}$.

In the following proposition, we show that the associated characterized fuzzy proximity space $\left(X, \varphi_{1,2} \cdot\right.$ int $\left._{\delta}\right)$ is characterized $F R_{2}$ space if the related fuzzy topological space $(X, \tau)$ is $F \varphi_{1,2}-R_{2}$ space.

\section{Proposition 3.5}

Let $(X, \tau)$ be a fuzzy topological space, $\varphi_{1}, \varphi_{2} \in O_{\left(L^{X}, \tau\right)}$ and is an fuzzy proximity on $X$. Then the associated characterized fuzzy proximity space $\left(X, \varphi_{1,2} \cdot\right.$.int $\left._{\delta}\right)$ is characterized $F R_{2}$-space if $(X, \tau)$ is $F \varphi_{1,2}-R_{2}$ space.

Proof: Let $x X$ and $\mu \in L^{X}$ with $\mathscr{N}_{\varphi_{1,2}}(x) \leq \dot{\mu}$. Because of Proposition 3.2, we have $\mu^{\prime} \bar{\delta} \rho$ and from (P5), there is $\rho \in L^{X}$ such that $\mu^{\prime} \bar{\delta} \rho$ and $\rho^{\prime} \bar{\delta} x_{1}$. Therefore Proposition 3.4 implies $\varphi_{1,2} \cdot \mathrm{cl}_{\delta} \mu^{\prime} \bar{\delta} \varphi_{1,2} \cdot \mathrm{cl}_{\delta} \rho$ and hence $\mathcal{N}_{\varphi_{1,2}}^{\delta}\left(\varphi_{1,2} \cdot \mathrm{cl}_{\delta} \rho\right) \leq\left(\varphi_{1,2} \cdot \mathrm{cl}_{\delta} \mu^{\prime}\right)^{\prime}$ and $\mathcal{N}_{\varphi_{1,2}}^{\delta}(x) \leq \dot{\rho}$ are hold. Hence, $\mathcal{N}_{\varphi_{1,2}}(x) \leq \dot{\mu}$ implies there is $\rho \in L^{X}$ such that $\mathcal{N}_{\varphi_{1,2}}^{\delta}(x) \leq \dot{\rho}$ and $\mathscr{N}_{\varphi_{1,2}}^{\delta}\left(\varphi_{1,2} . \mathrm{cl} \rho\right) \leq \dot{\mu}$ are hold. Since $\left(X, \varphi_{1,2} \cdot\right.$ int $\left._{\delta}\right)$ is $F \varphi_{1,2}-R_{2}$ space, then from Theorem 3.1 in Abd-Allah [12], we have $\left(X, \varphi_{1,2}\right.$.int $\left.{ }_{\delta}\right)$ is characterized $F R_{2}$-space.

The binary relation $<<$ on $L^{X}$ is said to be fuzzy topogeneous order on $X$ [23], if the following conditions are fulfilled:

(1) $\bar{\alpha} \ll \bar{\alpha}$ for all $\alpha \in\{0,1\}$.

(2) If $\mu \ll \eta$, then $\mu \leq \eta$ holds for all $\mu, \in L^{X}$.

(3) If ${ }_{1} \leq \mu \ll \eta \leq \eta_{1}$, then $\mu_{1} \ll \eta_{1}$ holds.

(4) If ${ }_{1} \ll \eta_{1}$ and $\mu_{2} \ll \eta_{2}$, then $\mu_{1} \wedge \mu_{2} \ll \eta_{1} \wedge \eta_{2}$ and $\mu_{1} \vee \mu_{2} \ll \eta_{1} \vee \eta_{2}$ are hold for all $\mu_{i}, \eta_{j} \in L^{X}$, where $i, j \in\{1,2\}$.

The fuzzy topogeneous order « is said to be fuzzy topogeneous structure if it fulfilled the condition:

(5) If $\ll \eta$, then there is $\sigma \in L^{X}$ such that $<<\sigma$ and $\sigma<<\eta$ are hold for all $\mu, \eta \in L^{X}$.

The fuzzy topogeneous structure $\ll$ is said to be fuzzy topogenous complementarily symmetric if it fulfilled the condition:

(6) If $<<\eta$, then $\eta^{\prime}<<\mu$ holds for all $\mu, \eta \in L^{X}$.

As shown in Katsaras [23], every fuzzy topogeneous structure $\ll$ is identify with the mapping $N: L^{X} \rightarrow P\left(L^{X}\right)$ such that $\eta \in N(\mu)$ if and only if $\mu<<\eta$ holds for all $\mu, \eta \in L^{X}$. The fuzzy topogeneous structures are 
Citation: Abd-Allah AS, Al-Khedhairi A (2017) The Relations between Characterized Fuzzy Proximity, Fuzzy Compact, Fuzzy Uniform Spaces and Characterized Fuzzy $T_{s}$-Spaces and Fuzzy $R_{k}$-Spaces. J Appl Computat Math 6: 337. doi: 10.4172/2168-9679.1000337

Page 9 of 15

classified by these mappings. As is easily seen, each fuzzy topogeneous order $N$ can be associated a fuzzy pre topology int ${ }_{N}$ on a set $X$ by $\operatorname{defining~}_{\operatorname{int}_{N}} \mu=\underset{\mu \in \mathcal{N}(\eta)}{\vee} \eta$ for all $\mu \in L^{X}$. In case of $N$ is fuzzy topogeneous structure, int ${ }_{N}$ is interior operator for fuzzy topology $\tau_{N}$ on $X$ associated toN. Obviously, there is an identification between the fuzzy proximity $\delta$ and the complementarily symmetric fuzzy topogenous structure $\ll$ on the same set $X$ given by:

$$
\mu \ll \eta^{\prime} \Leftrightarrow \mu \bar{\delta} \eta
$$

for all $\mu, \eta \in \in L^{X}$. If $\left\{\ll_{n}\right\}_{n=1}^{\infty}$ is a sequence of fuzzy topogenous structure on the set $X$ and $\left\{\prec_{n}\right\}_{n=1}^{\infty}$ is a sequence of fuzzy topogenous structure on $I_{L}$, then the fuzzy real function $f: X \rightarrow I_{L}$ is said to be associated with the sequence $\left\{\ll_{n}\right\}_{n=1}^{\infty}$ if and only if $\eta \prec_{n} \rho$ implies that $(\eta \circ f) \ll_{n+1}(\rho \circ f)$ holds for all $\eta, \rho \in L^{I_{L}}$ and $n \in \mathbb{Z}^{+}$, where $\mathbb{Z}^{+}$is the set of all positive integer numbers.

\section{Remark 3.2}

Given that $\left\{\ll_{n}\right\}_{n=1}^{\infty}$ and $\left\{\prec_{n}\right\}_{n=1}^{\infty}$ are two sequence of complementarily symmetric fuzzy topogenous structures $\ll$ and $\prec$ on $X$ and $I_{L}$, respectively. If $\delta$ and $\delta^{*}$ are two fuzzy proximities on $X$ and $I_{L}$ identified with $\delta$ and $\delta *$ by the equation (3.5), then the associated fuzzy real function $f:\left(X, \varphi_{1,2}\right.$. int $\left.\delta\right) \rightarrow\left(Y, \varphi_{1,2}\right.$.int $\left.{ }_{\delta^{*}}\right)$ with the complementarily symmetric fuzzy topogenous structures $\ll$ is $\varphi_{1,2} \psi_{1,2} \delta$ fuzzy continuous, because from (3.5) we get that $\eta \bar{\delta}^{*} \rho$ implies $(\eta \circ f) \bar{\delta}(\rho \circ f)$ for all $\eta, \rho \in L^{I_{L}}$.

\section{Lemma 3.2}

Consider $\ll_{n}$ for $n\{0,1, \ldots$,$\} are complementarily symmetric fuzzy$ topogenous structures on a set $X$. Then, for each $F, G \in P(X)$ such that $\chi_{F} \ll_{0} \chi_{G}$ there exists a fuzzy real function $f: X \rightarrow I_{L}$ associated with the sequence $\left\{\ll_{n}\right\}_{n=0}^{\infty}$ for which $f(x)=\overline{0}$ for all $x F$ and $f(y)=\overline{1}$ for all $y G^{\prime}[23]$.

Because of equation (3.5), Remark 3.2 and Lemma 3.2, we can easily deduce the following proposition.

\section{Proposition 3.6}

Let $\left(X, \varphi_{1,2} \cdot\right.$ int $\left._{\delta}\right)$ is a characterized fuzzy proximity space and $F, G \in P$ $(X)$ such that $\chi_{F} \bar{\delta} \chi_{G}$. If $\Phi$ is the family of all $_{1,2} \psi_{1,2} \delta$-fuzzy continuous mappings $f:\left(X, \varphi_{1,2} \cdot\right.$.nt $\left._{\delta}\right) \rightarrow\left(I_{L}, \psi_{1,2}\right.$. int $\left._{\delta^{*}}\right)$ for which $x \in X$ implies $\overline{0} \leq f(x) \leq \overline{1}$, then $\chi_{F}$ and $\chi_{G}$ are $\Phi$-separable.

Proof: Let « be a complementarily symmetric fuzzy topogenous structure identified with $\delta$. Because of (3.5), $\chi_{F} \bar{\delta} \chi_{G}$ implies that. Since $f \in \Phi$ is $\varphi_{1,2} \psi_{1,2} \delta$-fuzzy continuous, then because of Remark 3.2, we have that $f$ is associated with «. Hence, Lemma 3.2 implies that $\chi_{F}$ and $\chi_{G}$ are separated by $f$ and therefore $\chi_{F}$ and $\chi_{G}$ are $\Phi$-separable.

\section{Proposition 3.7}

Let $\left(X, \psi_{1,2}\right.$,int $\left._{\delta}\right)$ and $\left(Y, \psi_{1,2}\right.$. int $\left._{\delta^{*}}\right)$ are two characterized fuzzy proximity spaces. If the mapping $f:\left(X, \varphi_{1,2} \cdot\right.$ int $\left._{\delta}\right) \rightarrow\left(Y, \psi_{1,2} \cdot\right.$ int $\left._{\delta^{*}}\right)$ is ${ }_{1,2} \psi_{1,2} \delta$-fuzzy continuous, then the mapping $f:\left(X, \varphi_{1,2}\right.$.int $) \rightarrow\left(X, \psi_{1,2}\right.$.int $)$ is $\varphi_{1,2} \psi_{1,2}$-fuzzy continuous.

Proof: Similar to the proof of Proposition 11.2 in Gähler [13].

In the following we are going to show an important relation between the associated characterized fuzzy proximity space and the characterized $\mathrm{FR}_{3}$-space.

\section{Proposition 3.8}

Let $(X, \tau)$ be a fuzzy topological space and $\varphi_{1}, \varphi_{2} \in O_{\left(L^{X}, \tau\right)}$ such that $\varphi_{2} \geq 1_{{ }^{X}}$ is isotone and $\varphi_{1}$ is wfip with respect to $\varphi_{1} \mathrm{OF}(X)$, where $L$ is complete chain. If $(X, \tau)$ is a fuzzy normal topological space, then the binary relation $\delta$ on $X$ which is defined by:

$$
\mu \bar{\delta} \rho \Leftrightarrow \mathscr{N}_{\varphi_{1,2}}\left(\varphi_{1,2} \cdot \operatorname{cl} \mu\right) \leq\left(\varphi_{1,2} \cdot \operatorname{ci} \rho\right)^{\prime},
$$

for all $\mu, \rho \in L^{X}$ is a fuzzy proximity on $X$ and $(X, \delta)$ is a fuzzy proximity space. On other hand if $(X, \delta)$ is a fuzzy proximity space with $\delta$ fulfills (3.6), then the associated characterized fuzzy proximity space $\left(X, \psi_{1,2}\right.$, int $\left._{\delta}\right)$ is characterized $F R_{3}$-space.

Proof: Let $(X, \tau)$ is fuzzy normal topological space and $\delta$ a binary relation on $X$ defined by (3.6). Then, $\mu \bar{\delta} \rho$ implies $\mathcal{N}_{\varphi_{1,2}}\left(\varphi_{1,2} \cdot \operatorname{cl} \mu\right) \leq\left(\varphi_{1,2} \cdot \operatorname{ci} \rho\right)^{\prime}$ and from Lemma 3.1 part (1) we get $\mathcal{N}_{\varphi_{1,2}}\left(\varphi_{1,2} . \operatorname{cl} \rho\right) \leq\left(\varphi_{1,2} \cdot \operatorname{ci} \mu\right)^{\prime}$ and then $\rho \bar{\delta} \mu$. Hence, condition (P1) is fulfilled. For showing condition (P2), let $(\mu \vee \rho) \bar{\delta} \eta$ for a fixed fuzzy subsets $\mu, \rho, \eta \in L^{X}$. Then, $\quad \mathcal{N}_{\varphi_{1,2}}\left(\varphi_{1,2} \cdot \operatorname{cl}(\mu \vee \rho)\right) \leq\left(\varphi_{1,2} \cdot \operatorname{ci} \eta\right)^{\prime}$. Since $L$ is complete chain, $\varphi_{2} \geq 1_{L^{X}}$ is isotone and $\varphi_{1}$ is wfip with respect to $\varphi_{1} O F(X)$, then $\varphi_{1,2} \cdot \operatorname{cl}(\mu \vee \rho)=\varphi_{1,2} \cdot \operatorname{cl} \mu \vee \varphi_{1,2} \cdot \operatorname{cl} \rho$ and therefore $\mathcal{N}_{\varphi_{1,2}}\left(\varphi_{1,2} \cdot \mathrm{cl} \mu \vee \varphi_{1,2} \cdot \mathrm{cl} \rho\right) \leq\left(\varphi_{1,2} \cdot \mathrm{cl} \eta\right)^{\prime}$. Because of Lemma 3.1 part (3), we have $\mathcal{N}_{\varphi_{1,2}}\left(\varphi_{1,2} \cdot \operatorname{cl} \mu\right) \leq\left(\varphi_{1,2} \cdot \operatorname{cl} \eta\right)^{\prime}$ and $\mathscr{N}_{\varphi_{1,2}}\left(\varphi_{1,2} \cdot \operatorname{cl} \rho\right) \leq\left(\varphi_{1,2} \cdot \operatorname{cil} \eta\right)^{\prime}$ are hold and therefore $\mu \bar{\delta} \eta$ and $\rho \bar{\delta} \eta$. Thus, $(\mu \vee \rho) \bar{\delta} \eta$ implies $\mu \bar{\delta} \eta$ and $\rho \bar{\delta} \eta$. On the other hand let $\mu \bar{\delta} \eta$ and $\rho \bar{\delta} \eta$. Then from Lemma 3.1 we have tha the inequalities $\mathscr{N}_{\varphi_{1,2}}\left(\varphi_{1,2} \cdot \operatorname{cl} \mu\right) \leq\left(\varphi_{1,2} \cdot \operatorname{ci} \eta\right)^{\prime}$ and $\mathscr{N}_{\varphi_{1,2}}\left(\varphi_{1,2} \cdot \operatorname{cl} \rho\right) \leq\left(\varphi_{1,2} \cdot \operatorname{cl} \eta\right)^{\prime}$ are hold and therefore $\quad \mathscr{N}_{\varphi_{1,2}}\left(\varphi_{1,2} \cdot \operatorname{cl}(\mu \vee \rho)\right)=\mathcal{N}_{\varphi_{1,2}}\left(\varphi_{1,2} \cdot \operatorname{cl} \mu\right) \wedge \mathcal{N}_{\varphi_{1,2}}\left(\varphi_{1,2} \cdot \operatorname{cl} \rho\right) \leq\left(\varphi_{1,2} \cdot \operatorname{cl} \eta\right)^{\prime}$, that is, $\mu \bar{\delta} \eta$ and $\rho \bar{\delta} \eta$ imply $(\mu \vee \rho) \bar{\delta} \eta$. Hence, (P2) is fulfilled. Now, let $\mu, \rho \in L^{X}$ such that $\mu=\overline{0}$ or $\rho=\overline{0}$. Since $\mathcal{N}_{\varphi_{1,2}}(\overline{0})$ is the finest fuzzy filter on $X$ and from the fact $\varphi_{1,2} \cdot \operatorname{cl} \overline{0}=\overline{0}$, we get $\mathcal{N}_{\varphi_{1,2}}(\overline{0})=\mathcal{N}_{\varphi_{1,2}}\left(\varphi_{1,2} \cdot \operatorname{cl} \overline{0}\right) \leq\left(\varphi_{1,2} \cdot \operatorname{cl} \rho\right)^{\prime}$ holds for all $\rho \in L^{X}$. Thus, $\overline{0} \bar{\delta} \rho$ for all $\rho \in L^{X}$. Since $\mu=\overline{0}$ or $\rho=\overline{0}$, then we have $\mu \bar{\delta} \rho$, that is, (P3) is also fulfilled. Since $\mu \bar{\delta} \rho$ implies $\mathscr{N}_{\varphi_{1,2}}\left(\varphi_{1,2} . \operatorname{cl} \mu\right) \leq\left(\varphi_{1,2} . \mathrm{cl} \rho\right)^{\prime}$ which means by the inequality $\left(\varphi_{1,2} \cdot \operatorname{ci} \mu\right) \leq \mathcal{N}_{\varphi_{1,2}}\left(\varphi_{1,2} \cdot \operatorname{cl} \mu\right)$ that $\left(\varphi_{1,2} . \mathrm{cl} \mu\right) \leq\left(\varphi_{1,2} . \mathrm{d} \rho\right)^{\prime}$. Because of Proposition 2.1 and the fact that $\varphi_{1,2}$. cl is hull operator we get $\mu \leq \varphi_{1,2} \cdot \operatorname{cl} \mu \leq\left(\varphi_{1,2} \cdot \operatorname{cl} \rho\right)^{\prime} \leq \rho^{\prime}$. Thus, (P4) is fulfilled. Let $\mu, \rho \in L^{X}$ such that $\mu \bar{\delta} \rho$, then $\mathcal{N}_{\varphi_{1,2}}\left(\varphi_{1,2} \cdot \operatorname{cl} \mu\right) \leq\left(\varphi_{1,2} \cdot \operatorname{ci} \rho\right)^{\prime}$. Consider, $F=S_{0}\left(\varphi_{1,2} . \mathrm{cl} \mu\right)$, hence $F \in \varphi_{1,2} C(X)$ and therefore $\mathscr{N}_{\varphi_{1,2}}(F) \leq$ $\left(\varphi_{1,2} . \operatorname{ci} \rho\right)^{\prime}$ holds. Since $(X, \tau)$ is characterized fuzz normal space, then from Theorem 3.2 in Abd-Allah [12], there exists $\eta^{\prime} \in L^{X}$ with arbitrary choice such that $\mathcal{N}_{\varphi_{1,2}}(F) \leq \dot{\eta}^{\prime}$ and $\mathcal{N}_{\varphi_{1,2}}\left(\varphi_{1,2} \cdot \operatorname{cl} \eta^{\prime}\right) \leq\left(\varphi_{1,2} \cdot \operatorname{cl} \rho\right)^{\prime}$ are hold. Therefore, there exists $\in L^{X}$ such that $\mathscr{N}_{\varphi_{1,2}}\left(\varphi_{1,2} \cdot \operatorname{cl} \mu\right) \leq\left(\varphi_{1,2} \cdot \operatorname{cl} \eta\right)^{\prime}$ and $\mathscr{N}_{\varphi_{1,2}}\left(\varphi_{1,2} \cdot \operatorname{cl} \mu\right) \leq\left(\varphi_{1,2} \cdot \operatorname{ci} \eta\right)^{\prime}$, which means that $\mu \bar{\delta} \eta$ and $\eta^{\prime} \bar{\delta} \rho$. Hence, (P5) is also fulfilled. Consequently, $\delta$ is a fuzzy proximity on $X$.

Conversely, let $F_{1}, F_{2} \in \varphi_{1,2} C(X)$ such that $F_{1} \cap F_{2}=\emptyset$. Then, $F_{1} \subseteq F_{2}^{\prime}$ and therefore $\dot{\chi}_{F_{1}} \leq \dot{\chi}_{F_{2}^{\prime}}=\dot{\chi}_{F_{2}}^{\prime}$. Hence because of Lemma 3.1 part (1) we 
Citation: Abd-Allah AS, Al-Khedhairi A (2017) The Relations between Characterized Fuzzy Proximity, Fuzzy Compact, Fuzzy Uniform Spaces and Characterized Fuzzy $T_{s}$-Spaces and Fuzzy $R_{k}$-Spaces. J Appl Computat Math 6: 337. doi: 10.4172/2168-9679.1000337

have $\mathcal{N}_{\varphi_{1,2}}^{\delta}\left(\chi_{F_{2}}\right) \leq \dot{\chi}_{F_{1}}^{\prime}$. Since $F_{1}, F_{2} \in \varphi_{1,2} C(X)$, then $\mathscr{N}_{\varphi_{1,2}}^{\delta}\left(\varphi_{1,2} \cdot \mathrm{cl}_{\delta} \chi_{F_{2}}\right)=$ $\mathscr{N}_{\varphi_{1,2}}^{\delta}\left(\chi_{F_{2}}\right) \leq \dot{\chi}_{F_{1}}^{\prime}=\left(\varphi_{1,2} \cdot \mathrm{ci}_{\delta} \chi_{F_{1}}\right)^{\prime}$ and therefore $\chi_{F_{1}} \bar{\delta} \chi_{F_{2}}$. From (P5), there exists $\rho \in L^{X}$ such that $\mathcal{N}_{\varphi, 2}^{\delta}\left(\chi_{F_{2}}\right)=\mathcal{N}_{\varphi, 2}^{\delta}\left(F_{2}\right) \leq \dot{\rho}$ and $\mathscr{N}_{\varphi_{1,2}}^{\delta}(\rho) \leq \dot{\chi}_{F_{1}}^{\prime}=F_{1}^{\prime}$ are hold. Because of Lemma 3.1 part (1), we have $\mathcal{N}_{\varphi_{1,2}}^{\delta}\left(F_{1}\right) \leq \dot{\rho}^{\prime}$. Hence, $\mathscr{N}_{\varphi_{1,2}}^{\delta}\left(F_{1}\right)(\mu) \wedge \mathcal{N}_{\varphi_{1,2}}^{\delta}\left(F_{2}\right)(\eta) \geq \dot{\rho}^{\prime}(\mu) \wedge \dot{\rho}^{\prime}(\eta)$ holds for all. Consider $\eta=\chi_{F_{2}} \vee x_{1}=\rho$ and $\mu=\left(\chi_{F_{2}} \vee x_{1}\right)^{\prime}=\rho^{\prime}$ for all $x \in F_{1}^{\prime} \backslash F_{2}$, then we get $\sup (\mu \wedge)=0$ and $\mathcal{N}_{\varphi_{1,2}}^{\delta}\left(F_{1}\right)(\mu) \wedge \mathcal{N}_{\varphi_{1,2}}^{\delta}\left(F_{2}\right)(\eta) \geq 0$. Hence, there exist $\mu, \eta \in L^{X}$ such that $\mathcal{N}_{\varphi_{1,2}}^{\delta}\left(F_{1}\right)(\mu) \wedge \mathcal{N}_{\varphi_{1,2}}^{\delta}\left(F_{2}\right)(\eta) \geq \sup (\mu \wedge \eta)$, that is, the infimum $\mathcal{N}_{\varphi_{1,2}}^{\delta}\left(F_{1}\right) \wedge \mathcal{N}_{\varphi_{1,2}}^{\delta}\left(F_{2}\right)$ does not exists. Consequently, $\left(X, \psi_{1,2} \cdot\right.$ int $\left._{\delta}\right)$ is characterized $F R_{3}$-space.

In the following we are going to show an important relation between the associated characterized fuzzy proximity space $\left(X, \psi_{1,2}\right.$. int $_{\delta}$ ) by the fuzzy proximity $\delta$ defined by (3.6) and the associated characterized fuzzy space $\left(X, \psi_{1,2}\right.$.int) that introduced form the fuzzy normal topological space $(X, \tau)$.

\section{Proposition 3.9}

Let $(X, \tau)$ is a fuzzy normal topological space and $\varphi_{1}, \varphi_{2} \in O_{\left(I^{X}\right.}$ such that $\varphi_{2} \geq 1_{L^{X}}$ is isotone and $\varphi_{1}$ is wfip with respect to $\varphi_{1} O F\left(\mathcal{L}^{X}\right)$. If $\delta$ is the fuzzy proximity on $X$ defined by (3.6) and $L$ is a complete chain, then $\left(X, \varphi_{1,2}\right.$.int $)$ is finer than $\left(X, \varphi_{1,2}\right.$. int $\left.{ }_{\delta}\right)$. Moreover, $\left(X, \varphi_{1,2}\right.$. int $\left.\tau\right)$ $=\left(X, \varphi_{1,2}\right.$.int $\left.\delta_{\delta}\right)$ if and only if $\left(X, \varphi_{1,2}\right.$.int $)$ is characterized $F R_{4}$-space.

Proof: Let $(X, \tau)$ is fuzzy normal topological space and $\mu$ is $\varphi_{1,2} \delta$ fuzzy neighborhood for the point $x X$, then $x_{1} \bar{\delta} \mu^{\prime}$ and because of (3.6), we have $\mathscr{N}_{\varphi_{1,2}}\left(\varphi_{1,2} \cdot \mathrm{cl}_{\tau} x_{1}\right) \leq\left(\varphi_{1,2} \cdot \mathrm{cl}_{\tau} \mu^{\prime}\right)^{\prime}$. Therefore, $\dot{x} \leq \mathcal{N}_{\varphi_{1,2}}(x) \leq \mathcal{N}_{\varphi_{1,2}}\left(\varphi_{1,2} \cdot \mathrm{cl}_{\tau}\{x\}\right)=\mathscr{N}_{\varphi_{1,2}}\left(\varphi_{1,2} \cdot \mathrm{cl}_{\tau}\left(x_{1}\right)\right) \leq\left(\varphi_{1,2} \cdot \mathrm{cl}_{\tau} \mu^{\prime}\right)^{\prime} \leq \dot{\mu}$. Because of Proposition 2.1, we get $x_{1} \leq\left(\varphi_{1,2} \cdot \mathrm{cl}_{\tau} \mu^{\prime}\right)^{\prime} \leq \dot{\mu}$ and $\left(\varphi_{1,2} \cdot \mathrm{cl}_{\tau} \mu^{\prime}\right)^{\prime} \in \varphi_{1,2} O F(X)$. Then, $\mu$ is ${ }_{1,2}$-fuzzy neighborhood of $x$ and therefore the family $\left(\varphi_{1} O F(X)\right)_{\delta}$ is coarser than the family $\left(\varphi_{1} O F(X)\right)$, that is, $\left(X, \varphi_{1,2} \cdot\right.$ int $\left._{\tau}\right)$ is finer than $\left(X, \varphi_{1,2} \cdot\right.$ int $\left._{\delta}\right)$.

Now, let $\left(X, \varphi_{1,2}\right.$. int $\left._{\tau}\right)$ is characterized $F R_{4}$-space, $\mathscr{N}_{\varphi_{1,2}}$ and $\mathcal{N}_{\varphi_{1,2}}^{\delta}(x)$ denote for the $\varphi_{1,2}$-fuzzy neighborhood filters at $x$ in the characterized fuzzy space $\left(X, \varphi_{1,2}\right.$.int $\left.\tau_{\tau}\right)$ and in the associated characterized fuzzy proximity space $\left(X, \varphi_{1,2} \cdot\right.$ int $\left._{\delta}\right)$, respectively. Then, $\left(X, \varphi_{1,2}\right.$ int $\left.\tau_{\tau}\right)$ is characterized $F R_{3}$ and $F R_{1}$-space. Therefore, $\left(\varphi_{1,2} O F(X)\right)_{\delta} \subseteq\left(\varphi_{1,2} O F(X)\right)$ and $\mathcal{N}_{\varphi_{1,2}}(x) \leq \mathcal{N}_{\varphi_{2,2}}^{\delta}(x)$ holds for all $y$ $\neq x$ in $X$. Hence, $\mathcal{N}_{\varphi_{1,2}}(x) \leq \mathcal{N}_{\varphi_{1,2}}^{\delta}(x)^{\varphi_{1,2}}$ holds for all $x \in X$ and then $\mathscr{N}_{\varphi_{1,2}}^{\delta}(x) \geq \mathcal{N}_{\varphi_{1,2}}(x) \not z \dot{y}$ holds for all $y \neq x$ in $X$. Because of Lemma 2.1, we have that $\mathscr{N}_{\varphi_{1,2}}^{\delta}(x) \not \dot{y}$ holds for all $y \neq x$ in $X$ and therefore $\left(X, \varphi_{1,2} \cdot\right.$.nt $\left._{\delta}\right)$ is characterized $F T_{1}$-space. Because of Proposition 2.4 and Lemma 2.2, we get $\varphi_{1,2} \cdot \operatorname{cl}_{\tau}\left(x_{1}\right)=x_{1}$ for all $x \in X$ and therefore $x_{1} \in$ $\left(\varphi_{1,2} C F(X)\right)_{\delta}$ for all $x X$. Consider $\mu$ is the $\varphi_{1,2}$-fuzzy neighborhood of $x$ in $\left(X, \varphi_{1,2}\right.$.int $)$, then $\mu^{\prime} \leq x_{1}^{\prime}$ and since $x_{1}^{\prime} \in\left(\varphi_{1,2} O F(X)\right)_{\delta}$, then $x_{1}^{\prime}$ is a ${ }_{1,2}$-fuzzy neighborhood for every $y \in X$ such that $y_{1} \leq \mu^{\prime}$. Thus, $\mu^{\prime} \bar{\delta} x_{1}$ and hence $\mu$ is $\varphi_{1,2} \delta$-fuzzy neighborhood of $x$ in $\left(X, \varphi_{1,2} \cdot\right.$ int $\left._{\delta}\right)$. Thus, $\left(\varphi_{1,2} O F\right.$ $(X)) \subseteq\left(\varphi_{1,2} O F(X)\right)_{\delta}$, that is, $\mathscr{N}_{\varphi_{1,2}}^{\delta}(x) \leq \mathcal{N}_{\varphi_{1,2}}(x)$ holds for all $x \in X$ and therefore $\left(X, \varphi_{1,2}\right.$.int $\left.{ }_{\delta}\right)$ is finer than $\left(X, \varphi_{1,2}\right.$.int $)$. Consequently, $\left(X, \varphi_{1,2}\right.$. int $\left.\tau_{\tau}\right)$ is characterized $F R_{4}$-space implies that $\left(X, \varphi_{1,2} \cdot\right.$ int $\left._{\tau}\right)=\left(X, \varphi_{1,2} \cdot\right.$ int $\left._{\delta}\right)$.

Conversely, let $\left(X, \varphi_{1,2}\right.$.int $\left.\tau_{\tau}\right)=\left(X, \varphi_{1,2}\right.$.int $\left.{ }_{\delta}\right), x \in X$ and $\mu$ is $\varphi_{1,2}$-fuzzy neighborhood of $x$ in $\left(X, \varphi_{1,2} \text {.int }\right)_{\tau}$. Then, $\mu \in\left(\varphi_{1,2} O F(X)\right)_{\delta}$ and $x_{1 \leq} \mu$, this means that $\left(\varphi_{1,2} \cdot \mathrm{cl}_{\tau} x_{1}\right) \leq \mathscr{N}_{\varphi_{1,2}}\left(\varphi_{1,2} \cdot \mathrm{cl}_{\tau} x_{1}\right) \leq\left(\varphi_{1,2} \cdot \mathrm{cl}_{\tau} \mu^{\prime}\right)^{\prime} \leq \dot{\mu}$. Because of Proposition 2.1, we get $\varphi_{1,2} \cdot \mathrm{cl}_{\tau} x_{1} \leq \mu$ and therefore $\varphi_{1,2} \cdot \mathrm{cl}_{\tau} x_{1} \leq x_{1}$ holds for all $x \in X$. Thus, $\varphi_{1,2} \cdot \mathrm{cl}_{\tau} x_{1}=x_{1}$ for all $x \in X$. Hence, Proposition 2.4 implies that, $\left(X, \varphi_{1,2}\right.$.int $\left.\tau_{\tau}\right)$ is characterized $F R_{1}$-space. Because of Proposition 3.7, $\left.\left(X, \varphi_{1,2} \text {.int }\right)_{\delta}\right)$ is characterized $F R_{3}$-space and the hypothesis that $\left(X, \varphi_{1,2} \cdot\right.$ int $\left._{\tau}\right)=\left(X, \varphi_{1,2}\right.$. int $\delta$, implies that $\left.\left(X, \varphi_{1,2} \text {. int }\right)_{\tau}\right)$ is characterized $F R_{3}$-space. Consequently, $\left.\left(X, \varphi_{1,2} \text {. int }\right)_{\tau}\right)$ is characterized $\mathrm{FR}_{4}$-space.

In the following we are going to introduce some important relations joining our characterized $F R_{2 \frac{1}{2}}$ - spaces, characterized $F T_{2 \frac{1}{2}}{ }^{-}$ spaces and the associated characterized fuzzy proximity spaces.

\section{Proposition 3.10}

Let $(X, \tau)$ be an fuzzy topological space and $\varphi_{1}, \varphi_{2} \in O_{\left(L^{X}, \tau\right)}$. If $\delta$ is an fuzzy proximity on $X$, then the associated characterized fuzzy proximity space $\left(X, \varphi_{1,2} \cdot\right.$ int $\left._{\delta}\right)$ is characterized $F R_{2 \frac{1}{2}}$ - space.

Proof: Let $x X$ and $F \varphi_{1,2} C(X)$ such that $x \ddot{\mathrm{I}} F$. Since $\chi_{F^{\prime}}$ is ${ }_{1,2} \delta$-fuzzy neighborhood of $x$, then $x_{1} \bar{\delta} \chi_{F}$. Because of Proposition 3.2, we get that $x_{1}$ and $\chi_{\mathrm{F}}$ are $\Phi$-separated by the $\varphi_{1,2} \psi_{1,2} \delta$-fuzzy continuous mapping $f:\left(X, \varphi_{1,2} \cdot \mathrm{int} t_{\delta}\right) \rightarrow\left(I_{L}, \psi_{1,2} \cdot\right.$ int $\left.\delta^{*}\right)$ for which $\overline{0} \leq f(x) \leq \overline{1}$, that is, $f(x)=\overline{1}$ and $f(y)=\overline{1}$ for all $y \in F$. Consequently, $\left(X, \varphi_{1,2} \cdot\right.$ int $\left._{\delta}\right)$ is characterized $F R_{2 \frac{1}{2}}$ - space.

\section{Corollary 3.1}

Let $(X, \tau)$ be a fuzzy topological space, $\varphi_{1}, \varphi_{2} \in O_{\left({ }^{X}, \tau\right)}$ and $\delta$ is a fuzzy proximity on $X$. Then the associated characterized fuzzy proximity space $\left(X, \varphi_{1,2}\right.$. int $\left._{\delta}\right)$ is characterized $F T_{2 \frac{1}{2}}$ - space.

Proof : Immediately from Propositions 2.4 and 3.10.

Now, we introduce an example of an fuzzy proximity $\delta$ on a set $X$ and show that it is induces an associated characterized $F T_{2 \frac{1}{2}}$-space compatible with the related characterized fuzzy space.

\section{Example 3.1}

Let $L=\left\{0, \frac{1}{2}, 1\right\}, X=\{x, y\}$ and $=\left\{\overline{1}, \overline{0}, x_{1}, y_{1}\right\}$ is a fuzzy topology on $X$. Choose $\varphi_{1}=\operatorname{int}_{\tau}, \varphi_{2}=\mathrm{cl}_{\tau}, \psi_{1}=$ int $_{r}$, and $\psi_{2}=\mathrm{cl}$. Hence, $x \neq y$ and there is only two cases, the first is $x \ddot{I} F=\{y\} \in \varphi_{1,2} C(X)$ and the second is $y \ddot{I} F=\{x\} \in \varphi_{1,2} C(X)$. We shall consider the first case and the second case is similar. Consider the mapping $f:\left(X, \varphi_{1,2} \cdot\right.$ int $\left._{\tau}\right) \rightarrow$ $\left(I_{L}, \psi_{1,2}\right.$. int $\left._{I}\right)$ defined by $f(x)=\overline{1}$ and $f(y)=\overline{0}$, then $f$ is ${ }_{1,2} \psi_{1,2}$-fuzzy continuous and therefore $\left(X, \varphi_{1,2}\right.$.int $)$ is characterized $F R_{2 \frac{1}{2}}{ }^{-}$space and obviously $\left(X, \varphi_{1,2} \cdot\right.$ int $\left._{\tau}\right)$ is also characterized $F R_{1}$-space, that is, $\left(X, \varphi_{1,2} \cdot\right.$ int $\left._{\tau}\right)$ is characterized $F T_{3 \frac{1}{2}}$ - space. Now, consider $\delta$ is a binary relation on $L^{X}$ defied as follows:

$\mu \bar{\delta} \eta \Leftrightarrow \exists \varphi_{1,2} \psi_{1,2}$ - fuzzy continuous mapping $f:\left(X, \varphi_{1,2}\right.$.int $\left._{\tau}\right) \rightarrow$ $\left(I_{L}, \psi_{1,2}\right.$, int $\left._{I}\right) \ni f(x)=\overline{1}$ for all $x \in X$

with $x_{1} \leq \mu$ and $f(y)=\overline{0}$ for all $y_{1} \leq \eta$,

for all $\mu, \eta \in L^{X}$. Hence obviously, $\delta$ is a fuzzy proximity on $X$ and $\left(X, \varphi_{1,2}\right.$. int $\left.\tau_{\tau}\right)=\left(X, \varphi_{1,2 .}\right.$ int $\left._{\delta}\right)$, that is, the associated characterized fuzzy proximity space $\left(X, \varphi_{1,2}\right.$.int $\delta$ ) with $\delta$ is characterized $F T_{3 \frac{1}{2}}$ space and compatible with $\left(X, \varphi_{1,2}\right.$.int $\left.\tau_{\tau}\right)$.

\section{Some Relations between Characterized $F T_{s}$ and Characterized Fuzzy Compact Spaces}

The notion of $\varphi_{1,2}$-fuzzy compactness of the fuzzy filters and of 
Citation: Abd-Allah AS, Al-Khedhairi A (2017) The Relations between Characterized Fuzzy Proximity, Fuzzy Compact, Fuzzy Uniform Spaces and Characterized Fuzzy $T_{s}$-Spaces and Fuzzy $R_{k}$-Spaces. J Appl Computat Math 6: 337. doi: 10.4172/2168-9679.1000337

Page 11 of 15

the fuzzy topological spaces are introduced by Abd-Allah in [7] by means of the $\varphi_{1,2}$-fuzzy convergence in the characterized fuzzy spaces. Moreover, the fuzzy compactness in the characterized fuzzy spaces is also introduced by means of the $\varphi_{1,2}$-fuzzy compactness of the fuzzy filters and therefore it will be suitable to study here the relations between the characterized fuzzy compact spaces and some of our classes of separation axioms in the characterized fuzzy spaces.

Let $(X, \tau)$ be an fuzzy topological space, $F \subseteq X$ and $\varphi_{1}, \varphi_{2} \in O_{\left(L^{X}, \tau\right)}$. Then $x \in X$ is said to be $\varphi_{1,2}$-adherence point for the fuzzy filter $\mathcal{M}_{\text {on }} X[7]$, if the infimum $\mathcal{M}_{1,2} \wedge \mathcal{N}_{\varphi_{1,2}}(x)$ exists for all $\varphi_{1,2}$-fuzzy neighborhood filters $\mathcal{N}_{\varphi_{1,2}}(x)$ at $x \in X$. As shown in Abd-Allah [7], the point $x \in X$ is said to be $\varphi_{1,2}$-adherence point for the fuzzy filter $\boldsymbol{M}$ on $X$ if and

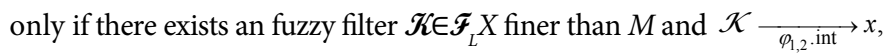

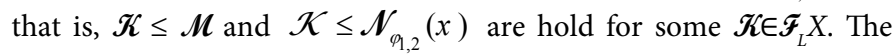
subset $F$ of $X$ is said to be $\varphi_{1,2}$-fuzzy closed with respect to $\varphi_{1,2}$ int if

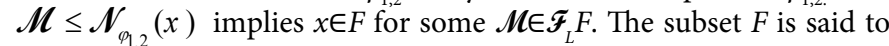
be $\varphi_{1,2}$-fuzzy compact [7], if every fuzzy filter on $F$ has a finer $\varphi_{1,2}$-fuzzy converging filter, that is, every fuzzy filter on $F$ has $\varphi_{1,2}$-adherence point in $F$. Moreover, the fuzzy topological space $(X, \tau)$ is said to be $\varphi_{1,2}$-fuzzy compact if $X$ is $\varphi_{1,2}$-fuzzy compact. More generally, the characterized fuzzy space $\left(X, \varphi_{1,2}\right.$.int) is said to be fuzzy compact space if the related fuzzy topological space $(X, \tau)$ is $\varphi_{1,2}$-fuzzy compact.

At first, in the following we shall benefit from these facts. Consider the fuzzy unit interval topological space $\left(I_{L}, \mathfrak{I}\right)$ be given and $\psi_{1}, \psi_{2} \in O_{\left(I_{L}, \mathfrak{s}\right)}$. Then:

(1) The usual topological space $\left(I, T_{I}\right)$ and the ordinary characterized usual space $\left(I, \psi_{1,2}\right.$. int $\left._{T_{I}}\right)$ on the closed unite interval $I=[0,1]$ are $\psi_{1,2}$ compact $T_{2}$ space and characterized compact $T_{2}$-space, respectively in the classical sense.

(2) The closed unite interval $I$ is identified with the fuzzy number $[0,1]^{\sim}$ in Gähler [24] defined by $[0,1]^{\sim}(\alpha)=0$ for all $\alpha \in I$ and $[0,1]^{\sim}(\alpha)$ $=0$ for all $\alpha \ddot{I} I$.

(3) The characterized fuzzy unite space $\left(I_{L}, \psi_{1}\right.$, int $\left.t_{\Im}\right)$ is up to an identification the characterized usual space $\varphi_{1}, \varphi_{2} \in O_{\left(L^{X}, \tau\right)}$ in the classical sense.

In the following proposition, we show that every $\varphi_{12}$-fuzzy compact subset in the characterized $F T_{2}$-space $\left(X, \varphi_{1,2}\right.$.int $\left.\tau_{\tau}\right)$ is $\varphi_{1,2}$-fuzzy closed with respect to the $\varphi_{1,2}$-interior operator $\varphi_{1,2}$. int $\tau_{\tau}$.

\section{Proposition 4.1}

Let a fuzzy topological space $(X, \tau)$ be fixed and $\varphi_{1}, \varphi_{2} \in O_{\left(L^{X}, \tau\right)}$. Then every $\varphi_{1,2}$-fuzzy compact subset of the characterized $F T_{2}$-space is ${ }_{1,2}$-closed.

Proof: Let $\left(X, \varphi_{1,2}\right.$.int $)$ is characterized $F T_{2}$-space and $F$ is $\varphi_{1,2}$-fuzzy compact subset of $X$. Then, for all $\boldsymbol{M} \in \mathscr{F}_{L} F$, there exists $\mathscr{K} \in \mathscr{F}_{L} F$ such that $\mathscr{K} \leq \mathcal{M}$ and $\mathcal{K} \leq \mathscr{N}_{\varphi_{1,2}}(x)$ for some $x \in F$. Since $\mathscr{K} \in \mathscr{F}_{L} F \leq F_{L} X$ and $\left(X, \varphi_{1,2} \cdot\right.$ int $\left._{\tau}\right)$ is characterized $F T_{2}$-space, then $\mathcal{K} \leq \mathscr{N}_{\varphi_{1,2}}(x)$ and $\mathcal{K} \leq \mathcal{N}_{\varphi_{1,2}}(y)$ imply that $x=y$. Therefore, $y \in F$ for some $\mathscr{K} \in \mathscr{F}_{L} F$. Hence, $F$ is $\varphi_{1,2}$-fuzzy closed with respect to ${ }_{1,2}$. int $\tau_{\tau}$.

\section{Proposition 4.2}

Let $\left(I_{L}, \mathfrak{I}\right)$ be a fuzzy unit interval topological space and $\psi_{1}, \psi_{2} \in O_{\left(L_{L} I_{\mathfrak{S}}\right)}$. Then the characterized fuzzy unit interval space
$\left(I_{L}, \psi_{1,2}\right.$.int $)$ is characterized fuzzy compact $F T_{2}$-space.

Proof: Let $\left(I, \psi_{1,2} \cdot\right.$.nt $\left._{T_{I}}\right)$ be an ordinary characterized usual space. Then, $\left(I, \psi_{1,2} \cdot\right.$. $\left._{T_{I}}\right)$ is characterized compact space in the classical sense, that is, every filter on $I$ has $\psi_{1,2}$-adherence point. Consider the mapping $f:\left(I, \psi_{1,2}\right.$. .nt $\left._{T_{I}}\right) \rightarrow\left(I_{L}, \psi_{1,2}\right.$. .nt $\left._{\mathfrak{\Im}}\right)$ defined by: $f(\alpha)=\tilde{\alpha}$ for all $\alpha \in I$, then it is easily to seen that $f$ is $\psi_{1,2} \psi_{1,2}$-fuzzy homeomorphism between $\left(I, \psi_{1,2} \cdot\right.$ int $\left._{T_{I}}\right)$ and $\left(I_{L}, \psi_{1,2} \cdot\right.$ int $\left._{\mathrm{I}}\right)$. Therefore, $\left(I_{L}, \psi_{1,2} \cdot\right.$ int $\left._{\mathrm{I}}\right)$ is characterized fuzzy compact space. Since $\left(I, T_{I}\right)$ is $\psi_{1,2} T_{2}$-space, then $\left(I, \psi_{1,2} \cdot \mathrm{i} n t_{T_{I}}\right)$ is characterized $F T_{2}$-space and therefore by using the same ${ }_{1,2} \psi_{1,2}$-fuzzy homeomorphism, we have for all $\tilde{\alpha}, \tilde{\beta} \in I_{L}$ such that $\tilde{\alpha} \neq \tilde{\beta}$, the infimum $\mathscr{N}_{\psi_{1,2}}(\tilde{\alpha}) \wedge \mathcal{N}_{\psi_{1,2}}(\tilde{\beta})$ does not exists. Consequently, $\left.\left(I_{L}, \psi_{1,2} \text {.int }\right)_{\mathrm{I}}\right)$ is characterized $F T_{2}$-space and therefore $\left(I_{L}, \psi_{1,2}\right.$. int $\left._{\mathrm{I}}\right)$ is characterized fuzzy compact $F T_{2}$-space.

Now, we are going to prove an important relation between the characterized compact $\mathrm{FT}_{2}$-spaces and the characterized $\mathrm{FT}_{4}$-spaces. For this reason at first, we give a new property for the characterized $F T_{2}$-spaces by using the $\varphi_{1,2}$-fuzzy neighborhood filters for the fuzzy subsets.

\section{Proposition 4.3}

Let $(X, \tau)$ be $\mathrm{n}$ fuzzy topological space and $\varphi_{1}, \varphi_{2} \in O_{\left(L^{X}, \tau\right)}$. Then every disjoint $\varphi_{1,2}$-fuzzy compact subsets $F_{1}$ and $F_{2}$ of in the characterized $F T_{2}$-space $\left(X, \varphi_{1,2}\right.$.int $\left.{ }_{\tau}\right)$ have two disjoint $\varphi_{1,2}$-fuzzy neighborhood filters $\mathcal{N}_{\varphi_{1,2}}\left(F_{1}\right)$ and $\mathcal{N}_{\varphi_{1,2}}\left(F_{2}\right)$ for which $F_{1}$ and $F_{2}$ are separated by them.

Proof: Let $F_{1}$ and $F_{2}$ are two $\varphi_{12}$-fuzzy compact subsets of the characterized $F T_{2}$-space $\left(X, \varphi_{1,2}\right.$.int $\left.\tau_{\tau}\right)$ such that $F_{1} \cap F_{2}=\emptyset$. Then, for all $\boldsymbol{M}_{i} \in \mathcal{F}_{L} F_{i}$ there exists $\mathscr{K}_{i} \in \mathcal{F}_{L} F_{i}$ such that $\mathscr{K}_{i} \leq \mathcal{M}_{i}$ and $\mathcal{K}_{i} \leq \mathcal{N}_{\varphi_{12}}\left(x_{i}\right)$ for some $x_{i} F_{i}$, where $i \in\{1,2\}$. Since $\mathscr{F}_{L} F_{i} \leq \mathscr{F}_{L} X$ for all $i \in\{1,2\}$, then we can say that $\mathcal{K}_{i} \leq \mathcal{N}_{\varphi_{1,2}}\left(x_{i}\right) \leq \mathcal{N}_{\varphi_{1,2}}\left(F_{i}\right)$ and therefore there is $\mathcal{K}=\mathcal{K}_{1} \wedge \mathcal{K}_{2} \in \mathscr{F}_{L} X$ such that $\mathcal{K} \leq \mathcal{N}_{\varphi_{1,2}}\left(x_{i}\right)$ for some $x_{i} F_{i}$. Since $\left(X, \varphi_{1,2}\right.$. int $\left._{\tau}\right)$ is characterized $F T_{2}$-space, then $x_{1}=x_{2}$ which contradicts $F_{1} \cap F_{2}=\emptyset$. Hence, for every $\mathcal{L} \in \mathcal{F}_{L} X$ we get $\mathcal{L} \leq \mathcal{N}_{\varphi_{1,2}}\left(F_{1}\right)$ or $\mathcal{L} \not \mathscr{N}_{\varphi_{1,2}}\left(F_{2}\right)$ which means that the infimum $\mathcal{N}_{\varphi_{1,2}}\left(F_{1}\right) \wedge \mathcal{N}_{\varphi_{1,2}}\left(F_{2}\right)$ does not exists and therefore $F_{1}$ and $F_{2}$ can be separated by two disjoint $\varphi_{1,2}$-fuzzy neighborhood filters.

Secondly, the notion of the fuzzy compactness for the characterized fuzzy spaces fulfills the following property which will be also used in the prove of this important result which given in Proposition 4.4.

\section{Lemma 4.1}

Let $(X, \tau)$ be a fuzzy topological space and $\varphi_{1}, \varphi_{2} \in O_{\left(L^{X}, \tau\right)}$. Then every $\varphi_{1,2}$-fuzzy closed subset of the characterized fuzzy compact space $\left(X, \varphi_{1,2}\right.$. int $\left._{\tau}\right)$ is $\varphi_{1,2}$-fuzzy compact.

Proof: Let $F$ is $\varphi_{1,2}$-fuzzy closed subset of the characterized fuzzy compact space $\left(X, \varphi_{1,2}\right.$.int $\left.\tau_{\tau}\right)$ and let $\boldsymbol{M} \in \mathcal{F}_{L} F$. Then, $\boldsymbol{M} \leq \mathscr{N}_{\varphi_{1,2}}(x)$ implies that $x \in F$. Since $\mathcal{F}_{L} F \leq \mathscr{F}_{L} X$, then $\mathcal{M}_{\mathcal{F}} \mathscr{F}_{L} X$ and hence there exists $\mathscr{K} \in \mathcal{F}_{L} X$ such that $\mathscr{K} \leq \mathcal{M}$ and $\mathcal{K} \leq \mathscr{N}_{\varphi_{1,2}}(x)$. Since $\boldsymbol{M} \in \mathscr{F}_{L} F$ and $\mathscr{K} \leq \mathcal{M}$, then $\mathscr{K} \in \mathcal{F}_{L} F$. Thus, for all $M \in F_{L} F$ we get $\mathscr{K} \leq \mathcal{M}$ such that $\mathcal{K} \leq \mathscr{N}_{\varphi_{1,2}}(x)$. Therefore, $x \in F$ is $\varphi_{1,2}$-adherence point of $\mathcal{M}$, that is, $F$ is $\varphi_{1,2}$-fuzzy compact. 
Citation: Abd-Allah AS, Al-Khedhairi A (2017) The Relations between Characterized Fuzzy Proximity, Fuzzy Compact, Fuzzy Uniform Spaces and Characterized Fuzzy $T_{s}$-Spaces and Fuzzy $R_{k}$-Spaces. J Appl Computat Math 6: 337. doi: 10.4172/2168-9679.1000337

Page 12 of 15

\section{Proposition 4.4}

Let $(X, \tau)$ be an fuzzy topological space and $\varphi_{1}, \varphi_{2} \in O_{\left(L^{X}, \tau\right)}$. Then every characterized fuzzy compact $F T_{2}$-space $\left(X, \varphi_{1,2}\right.$.int $\left.{ }_{\tau}\right)$ is characterized $\mathrm{FT}_{4}$-space.

Proof: Follows directly from Lemma 4.1 and Proposition 4.3 .

One of the application of Proposition 4.4, we have more generally the following result to the characterized fuzzy unit interval space.

\section{Proposition 4.5}

Let $\left(I_{L}, \mathfrak{I}\right)$ be an fuzzy unit interval topological space and $\psi_{1}, \psi_{2} \in O_{\left(L_{L} I_{\mathfrak{I}}\right)}$. Then the characterized fuzzy unit interval space $\left(I_{L}, \psi_{1,2}\right.$. int $\left._{\mathrm{I}}\right)$ is characterized $F T_{3 \frac{1}{2}}{ }^{-}$space.

Proof: Because of Proposition 4.2, the characterized fuzzy unit interval space $\left(I_{L}, \psi_{1,2}\right.$. int $\left._{\mathrm{T}}\right)$ is characterized fuzzy compact $F R_{2}$-space. Therefore from Proposition 4.4, we get $\left(I_{I}, \psi_{1,2}\right.$.int $)$ is characterized $F R_{4}$ space. Hence, Proposition 4.6 in Abd-Allah [11] gives us that, $\left(I_{L}, \psi_{1,2}\right.$. int $_{\mathrm{I}}$ ) is characterized $F T_{3 \frac{1}{2}}$ - space.

The $\varphi_{1,2}$-fuzzy compactness in the characterized fuzzy spaces is applied to fulfilled the Generalized Tychonoff Theorem [11] and from (2) in Proposition 2.6, the characterized fuzzy product space of the characterized $F R_{2}$-spaces is also characterized $F R_{2}$-space. Hence, by means of Propositions 4.2 and 4.4, the following result goes clear.

\section{Proposition 4.6}

Let $\left(I_{L}, \mathfrak{I}\right)$ be a fuzzy unit interval topological space and $\psi_{1}, \psi_{2} \in O_{\left(L, I_{L}, \mathfrak{s}\right)}$. Then the characterized fuzzy cube is characterized $F T_{2}$-space and it is characterized $F T_{4}$-space.

Proof: Since the characterized fuzzy cube is product of copies of the characterized fuzzy unit interval space $\left(I_{L}, \psi_{1,2} \cdot\right.$ int $\left._{\Im}\right)$ and by means of Proposition $4.2,\left(I_{L}, \psi_{1,2}\right.$. int $\left._{\mathfrak{J}}\right)$ is characterized fuzzy compact $F T_{2}$-space. Then because of Proposition 2.6, part (3) and Generalized Tychonoff Theorem in Abd-Allah [11], it follows that, the characterized fuzzy cube is characterized $\mathrm{FT}_{2}$-space. Moreover, Proposition 5.1, it follows that the characterized fuzzy cube is characterized $\mathrm{FT}_{4}$-space.

\section{Lemma 4.2}

Let $(X, \tau)$ and $(X, \sigma)$ are two fuzzy topological spaces such that $\tau$ is finer than $\sigma$ If $\varphi_{1}, \varphi_{2} \in O_{\left(L^{X}, \tau\right)}, \psi_{1}, \psi_{2} \in O_{\left(L^{X}, \sigma\right)}$ and $\left(X, \psi_{1,2}\right.$. int $\left.{ }_{\sigma}\right)$ is characterized fuzzy compact space, then $\left(X, \varphi_{1,2}\right.$.int $\left.{ }_{\tau}\right)$ s also characterized fuzzy compact space.

Proof: Let $\mathcal{N}_{\varphi_{1,2}}(x)$ and $\mathcal{N}_{\psi_{1,2}}(x)$ are the $\varphi_{1,2}$-fuzzy neighborhood and $\psi_{1,2}$-fuzzy neighborhood at $x$ with respect to $\psi_{1,2}$. int ${ }_{\tau}$ and $\psi_{1,2}$. int ${ }_{\sigma}$ respectively. Since $\tau$ is finer than $\sigma$, then $\mathscr{N}_{\psi_{1,2}}(x) \leq \mathcal{N}_{\varphi_{1,2}}(x)$ for all $x \in X$. Because of $\psi_{1,2}, i n t_{\sigma}$, is characterized fuzzy compact space, then for all $\mathcal{M} \in \mathcal{F}_{L} X$, there exists $\mathscr{K} \in \mathscr{F}_{L} X$ such that $\mathscr{K} \leq \mathcal{M}_{\text {and }} \mathcal{K} \leq \mathcal{N}_{\psi_{1,2}}(x)$ for all $x X$. Therefore $\mathcal{K} \leq \mathcal{N}_{\varphi_{1,2}}(x)$ for all $x X$. Consequently, $\left(X, \varphi_{1,2}\right.$. int $_{\tau}$ ) is characterized fuzzy compact space.

\section{Proposition 4.7}

Let $(X, \tau)$ and $(X, \sigma)$ are two fuzzy topological spaces such that is finer than $\varphi_{1}, \varphi_{2} \in O_{\left(L^{X}, \tau\right)}$ and $\psi_{1}, \psi_{2} \in O_{\left(L^{X}, \sigma\right)}$. If $\left(X, \psi_{1,2} \cdot\right.$.int $\left._{\sigma}\right)$ is characterized fuzzy compact space and $\left(X, \varphi_{1,2}\right.$.int $\left.\tau_{\tau}\right)$ is characterized $F T_{2}$ space, then $\left(X, \varphi_{1,2}\right.$.int $\left.{ }_{\tau}\right)$ and $\left(X, \psi_{1,2}\right.$.int $\left.{ }_{\sigma}\right)$ are $\varphi_{1,2} \psi_{1,2}$-fuzzy isomorphic.

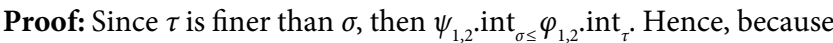
of Proposition 2.5, $\left(X, \psi_{1,2}\right.$.int $\left.{ }_{\sigma}\right)$ is characterized $F T_{2}$-space. From Lemma 4.2 , we have $\left(X, \varphi_{1,2}\right.$. int $\left._{\tau}\right)$ is characterized fuzzy compact space. Hence, we can find the identity mapping $i d_{X}:\left(X, \varphi_{1,2}\right.$.int $\left.\tau_{\tau}\right) \rightarrow\left(X, \psi_{1,2}\right.$.int $\left.{ }_{\sigma}\right)$ which is bijective $\varphi_{1,2} \psi_{1,2}$-fuzzy continuous and its inverse is $\varphi_{1,2} \psi_{1,2}$-fuzzy continuous, that is, $i d_{X}$ is $\varphi_{1,2} \psi_{1,2}$-fuzzy isomorphism. Consequently, $\left(X, \varphi_{1,2}\right.$.int $\left.\tau_{\tau}\right)$ and $\left(X, \psi_{1,2}\right.$.int $\left.{ }_{\sigma}\right)$ are $\varphi_{1,2} \psi_{1,2}$-fuzzy isomorphic.

\section{Proposition 4.8}

Let $(X, \tau)$ be a fuzzy topological spaces and $\varphi_{1}, \varphi_{2} \in O_{\left(L^{X}, \tau\right)}$. Then every characterized fuzzy compact space $\left(X, \varphi_{1,2}\right.$. int $\left._{\tau}\right)$ is characterized $F T_{2}$-space if and only if it is characterized $F T_{3 \frac{1}{2}}$ - space.

Proof: Let $\left(X, \varphi_{1,2}\right.$.int $)$ is characterized fuzzy compact $F T_{2}$-space. Because of Proposition 4.4 we have $\left(X, \varphi_{1,2}\right.$.int $)$ is characterized $F T_{4}$ space and therefor Proposition 4.6 in Abd-Allah S [11], implies that $\left(X, \varphi_{1,2}\right.$. int $\left._{\tau}\right)$ is characterized $F T_{3 \frac{1}{2}}$ - space. Conversely, let $\left(X, \varphi_{1,2}\right.$. int $\left._{\tau}\right)$ is characterized $F T_{3 \frac{1}{2}}$ - space, then because of Proposition 3.2 in AbdAllah [11] and part (1) of Proposition 2.6, it follows that $\left(X, \varphi_{1,2}\right.$. int $\left._{\tau}\right)$ is characterized fuzzy compact $F T_{2}$-space.

From Lemma 4.2 and Corollary 3.3 in [22], we can prove the following result.

\section{Proposition 4.9}

Let $(X, \tau)$ and $(X, \sigma)$ are two fuzzy topological spaces such that $\tau$ is finer than $\sigma, \varphi_{1}, \varphi_{2} \in O_{\left(L^{X}, \tau\right)}$ and $\psi_{1}, \psi_{2} \in O_{\left(L^{X}, \sigma\right)}$. If $\left(X, \varphi_{1,2}\right.$.int $)$ is characterized fuzzy compact space and $\left(X, \psi_{1,2}\right.$.int $\left.t_{\sigma}\right)$ is characterized $F T_{3 \frac{1}{2}}$-space, then $\left(X, \varphi_{1,2}\right.$.int $\left.\tau_{\tau}\right)$ and $\left(X, \psi_{1,2}\right.$.int $\left.\sigma_{\sigma}\right)$ are $\varphi_{1,2} \psi_{1,2}$-fuzzy isomorphic.

Proof: Follows directly from Corollary 3.3 in [22] and Lemma 4.2 similar to the proof of Proposition 4.7.

\section{Some Relations Between Characterized $F T_{s}$, Characterized $F R_{k}$ and Characterized Fuzzy Uniform Spaces}

In this section, we are going to investigate and study the relations between the characterized $F T_{s}$-spaces, the characterized $F T_{k}$-spaces and the characterized fuzzy uniform spaces presented in Abd-Allah [12]. For this, we applied the notion of homogeneous fuzzy filter at the point and at the fuzzy set which is defined by (2.1), the superior principal fuzzy filter $[\mu]$ generated by $\mu \in L^{X}$ and the $\varphi_{1,2}$-fuzzy neighborhoods at the fuzzy set $\mu$ which is defined by (3.1) in the characterized fuzzy space $\left(X, \varphi_{1,2}\right.$.int $\left.\tau_{\tau}\right)$. Specially, the relation between the separated fuzzy uniform spaces, the associated characterized fuzzy uniform $F T_{s}$-spaces, the associated characterized uniform $F R_{2 \frac{1}{2}}$ - space and the $F \varphi_{1,2} T_{s}$ space which introduced by Abd-Allah and Abd-Allah et al. in $[8,11]$ are investigated for all $s \in\left\{0,1,3 \frac{1}{2}\right\}$.

By the fuzzy relation on the set $X$, we mean the mapping $R: X \times X$ $\rightarrow L$, that is, any fuzzy subset of $X \times X$. For each fuzzy relation $R$ on $X$, the inverse $R^{-1}$ of $R$ is the fuzzy relation on $X$ defined by $R^{-1}(x, y)=R$ $(y, x)$ for all $x, y \in X$. Let $\boldsymbol{U}$ be a fuzzy filer on $X \times X$. The inverse $\boldsymbol{U}^{-1}$ of $\boldsymbol{U}$ is a fuzzy filter on $X \times X$ defined by $\boldsymbol{U}^{-1}(R)=\boldsymbol{U}\left(R^{-1}\right)$ for all $R L^{X \times X}$. The composition $R_{1} \circ R_{2}$ of two fuzzy relations $R_{1}$ and $R_{2}$ on the set $X$ is a fuzzy relation on $X$ defined by:

$$
\left(R_{1} \circ R_{2}\right)(x, y)=\bigvee_{z \in X}\left(R_{2}(x, z) \wedge R_{1}(z, y)\right)
$$

for all $x, y \in X$. For each pair $(x, y)$ of elements $x$ and $y$ of $X \times X$, the mapping $(x, y)^{\prime}: L^{X \times X} \rightarrow X$ defined by: $(x, y)^{\cdot}(R)=R(x, y)$ for all $R \in X \times X$ is a homogeneous fuzzy filter on $X \times X$. Let $\boldsymbol{U}$ and $\boldsymbol{V}$ are fuzzy filers on 
Citation: Abd-Allah AS, Al-Khedhairi A (2017) The Relations between Characterized Fuzzy Proximity, Fuzzy Compact, Fuzzy Uniform Spaces and Characterized Fuzzy $T_{s}$-Spaces and Fuzzy $R_{k}$-Spaces. J Appl Computat Math 6: 337. doi: 10.4172/2168-9679.1000337

Page 13 of 15

$X \times X$ such that $(x, y) \cdot \leq \mathcal{U}$ and $(y, z) \cdot V V$ hold for some $x, y, z \in X$. Then the composition $\boldsymbol{V}_{\mathrm{o}} \boldsymbol{U}$ of $\boldsymbol{V}_{\text {and }} \boldsymbol{U}$ is a fuzzy filter [13] on $X \times X$ defined by:

$$
(\mathcal{V} \circ \mathcal{U})(R)=\bigvee_{R_{2} \circ R_{1} \leq R}\left(\mathcal{U}\left(R_{1}\right) \wedge \mathcal{U}\left(R_{2}\right)\right)
$$

for all $R \in L^{X \times X}$.

By the fuzzy uniform structure $\mathcal{U}$ on a set $X[13]$, we mean a fuzzy filter on $X \times X$ such that the following axioms are fulfilled:

(U1) $(x, x) \cdot \leq \boldsymbol{U}$ for all $x \in X$.

(U2) $\boldsymbol{U}=\boldsymbol{U}^{-1}$

(U3) $\boldsymbol{U}_{\mathrm{o}} \boldsymbol{u} \leq \boldsymbol{U}$

The pair $(X, \mathscr{U})$ is called fuzzy uniform space. The fuzzy uniform structure $\mathcal{U}$ [13] on a set $X$ is said to be separated if for all $x, y \in X$ with $x \ddot{\mathrm{I}} y$ there is $R \in L^{X \times X}$ such that $\boldsymbol{U}(R)=1$ and $R(x, y)=0$. In this case the fuzzy uniform space $(X, \mathscr{U})$ is called separated fuzzy uniform space. Let $\boldsymbol{U}$ is a fuzzy uniform structure on a set $X$ such that $(x, x) \leq \boldsymbol{U}$ holds for all $x \in X$ and let $\boldsymbol{M} \in \mathcal{F}_{L} X$ then the mapping $\mathcal{U}[\boldsymbol{M}]: L^{X} \rightarrow L$ which is defined by:

$$
\boldsymbol{U}[\boldsymbol{M}](\mu)=\underset{R(\eta) \leq \mu}{\bigvee}(\mathcal{U}(R) \wedge \boldsymbol{M}(\eta))
$$

for all $\mu \in L^{X}$ is a fuzzy filter on $X$, called the image of $\boldsymbol{M}$ with respect to the fuzzy uniform structure $\mathcal{U}[13]$, where $\eta, R[\eta] \in L^{X}$ such that

$$
R[\eta](x)=\bigvee_{y \in X}(\eta(y), R(y, x)) .
$$

Each fuzzy uniform structure $\boldsymbol{U}$ on the set $X$ is associated a stratified fuzzy topology $\tau_{u}$ on $X$. Consider $\varphi_{1}, \varphi_{2} \in O_{\left(L^{X}, \tau_{u}\right)}$, then the set of all $\varphi_{1,2}$-open fuzzy subsets of $X$ related to $\tau_{U}$ forms a base for an characterized stratified fuzzy space on $X$ generated by the $\varphi_{1,2}$-interior operator with respect to $\tau_{u}$ denoted by $\varphi_{1,2} \cdot$ int $_{u}$ and $\left(X, \varphi_{1,2}\right.$.int $\left.{ }_{u}\right)$ is the related stratified characterized fuzzy space. In this case, $\left(X, \varphi_{1,2}\right.$.int $\left.{ }_{u}\right)$ will be called the associated characterized fuzzy uniform space [12] which is stratified. The related $\varphi_{1,2}$-interior operator $\varphi_{1,2} \cdot$ int $_{U}$ is given by:

$$
\left(\varphi_{1,2} \cdot \text { int }_{U} \mu\right)(x)=\mathcal{U}[\dot{\mathrm{x}}](\mu)
$$

for all $x \in X$ and $\mu \in L^{X}$. The fuzzy set $\mu$ is said to be $\varphi_{1,2} \boldsymbol{u}$-fuzzy neighborhood of $x \in X$ in the associated characterized fuzzy uniform space $\left(X, \varphi_{1,2}\right.$, int $\left._{\psi}\right)$, provided $\mathcal{U}[\dot{x}] \leq \dot{\mu}$. Because of $(2.1),(3.1)$ and (5.1) we have that

$$
\mathcal{U}[\dot{x}]=\mathcal{N}_{\varphi_{1,2}}(x) \text { and } \mathcal{U}[\dot{\mu}]=\mathcal{N}_{\varphi_{1,2}}(\mu)
$$

for all $x \in X$ and $\mu \in L^{X}$. In this case $\mathcal{N}_{\varphi_{1,2}}(x)$ and $\mathcal{N}_{\varphi_{1,2}}(\mu)$ are the $\varphi_{1,2}$-fuzzy neighborhood filters of the associated characterized fuzzy uniform space $\left(X, \varphi_{1,2} \cdot\right.$ int $\left._{u}\right)$ at $x$ and $\mu$, respectively.

\section{Proposition 5.1}

Let $X$ be a non-empty set, $U$ is a fuzzy uniform structure on $X$ and $\varphi_{1}, \varphi_{2} \in O_{\left(L^{X}, \tau_{u}\right)}$. Then the fuzzy uniform space $(X, U)$ is separated if and only if the associated characterized fuzzy uniform space $\left(X, \varphi_{1,2}\right.$. int $\left._{u}\right)$ is characterized $F T_{0}$-space.

Proof: Let $(X, \mathcal{U})$ is separated and let $x, y \in X$ such that $x \neq y$. Then, there exists $R \in L^{X \times X}$ such that $\mathcal{U}(R)=1$ and $R(x, y)=0$. Consider $\mu=$ $R\left[y_{1}\right]$ for which

$$
\begin{aligned}
& \mu(x)=R\left[y_{1}\right](x)=\bigvee_{z \in X} R(z, x) \wedge y_{1}(z)=0 \text { and } \\
& \left(\varphi_{1,2} \cdot \text {.nt }_{\mathcal{U}} \mu\right)(y)=\mathcal{U}[\dot{y}](\mu)=\underset{R(\eta) \leq \mu}{\bigvee} \boldsymbol{U}(R) \wedge \eta(y)=1
\end{aligned}
$$

for all $\eta \in L^{X}$. Hence, there exists $\mu \in L^{X}$ and $\alpha \in L_{0}$ such that $\mu(x)<\alpha$ $\leq\left(\varphi_{1,2} \cdot\right.$ int $\left._{u}{ }^{\prime}\right)(y)$, that is, $\left(X, \varphi_{1,2} \cdot\right.$.nt $\left._{u}\right)$ is characterized $F T_{0}$-space.

Conversely, let $\left(X, \varphi_{1,2} \cdot\right.$ int $\left._{U}\right)$ is characterized $F T_{0}$-space and let $x \neq y$ in $X$. Then, there exists $\mu \in L^{X}$ and $\alpha \in L_{0}$ such that $\mu(x)<\alpha \leq\left(\varphi_{1,2}\right.$.int $\left.{ }_{u} \mu\right)$ (y). This means that $\underset{R(\eta) \leq \mu}{\bigvee} \boldsymbol{U}(R) \wedge \eta(y)>\mu(x)$ holds for all $\eta \in L^{X}$. Hence, there is $R \in L^{X \times X}$ for which $R(x, y)=\left(\varphi_{1,2}\right.$.int $\left.u\right)(x)$, if $x=y$ and $R(x, y)=\mu(x)$, if $x \neq y$ such that $R(x, y)=0$ and $\boldsymbol{U}(R)=1$. Thus, $(X, \boldsymbol{U})$ is separated.

\section{Corollary 5.1}

Let $X$ be a non-empty set, $\boldsymbol{U}$ is a fuzzy uniform structure on $X$ and $\varphi_{1}, \varphi_{2} \in O_{\left(L^{X}, \tau_{\mathcal{L}}\right)}$. Then the fuzzy uniform space $(X, \mathcal{U})$ is separated if and only if the associated stratified fuzzy topological space $\left(X, \tau_{u}\right)$ is $F \varphi_{1,2}-T_{0}$ space.

Proof: Immediate from Proposition 5.1 and Theorem 2.1 in AbdAllah [8].

\section{Proposition 5.2}

Let $X$ be a non-empty set, $\mathcal{U}$ is a fuzzy uniform structure on $X$ and $\varphi_{1}, \varphi_{2} \in O_{\left(L^{X}, \tau_{\mathcal{U}}\right)}$. Then the fuzzy uniform space $(X, \mathcal{U})$ is separated if and only if the associated characterized fuzzy uniform space $\left(X, \varphi_{1,2}\right.$. int $_{u}$ ) is characterized $F T_{1}$-space.

Proof: Let $(X, \mathcal{U})$ is separated and let $x, y \in X$ such that $x \neq y$. Then, there exists $R_{1}, R_{2} \in L^{X \times X}$ such that $\mathcal{U}\left(R_{i}\right)=1$ and $R_{i}(x, y)=0$ for all $\mathrm{i} \in\{1.2\}$. Consider $\mu=R\left[y_{1}\right]$ and $\eta=\mathrm{R}\left[x_{1}\right]$, then we have $\mu(x)=R_{1}\left[y_{1}\right](x)=$ $\bigvee_{z \in X}\left(R_{1}(z, x) \wedge y_{1}(z)\right)=0$ and $\eta(y)=R_{2}\left[x_{1}\right](y)=\bigvee_{z \in X}\left(R_{2}(z, y) \wedge x_{1}(z)\right)=0$. Moreover, $\quad\left(\varphi_{1,2} \cdot \operatorname{int}_{u} \mu\right)(y)=\mathcal{U}[\dot{y}](\mu)=\bigvee_{R_{1}(\rho) \leq \mu}\left(\mathcal{U}\left(R_{1}\right) \wedge \rho(y)\right)=1 \quad$ and $\left(\varphi_{1,2} \cdot\right.$.nt $\left._{\mathcal{u}} \eta\right)(x)=\boldsymbol{U}[\dot{x}](\eta)=\bigvee_{R_{1}(\rho) \leq \eta}\left(\mathcal{U}\left(R_{2}\right) \wedge \rho(x)\right)=1$ for all $\rho \in L^{X}$. Hence, there exists $\mu, \eta L^{X}$ and $\alpha, \beta L_{0}$ such that $\mu(x)<\alpha \leq\left(X, \varphi_{1,2}\right.$.int $\left.{ }_{u} \mu\right)$ $(y)$ and $\eta(y)<\beta \leq\left(\varphi_{1,2}\right.$.int $\left._{\eta}\right)(x)$ are hold. Consequently, $\left(X, \varphi_{1,2}\right.$.int $\left.{ }_{u}\right)$ is characterized $F T_{1}$-space.

Conversely, let $\left(X, \varphi_{1,2} \cdot\right.$ int $\left._{u}\right)$ is characterized $F T_{1}$-space and let $x$ $\neq y$ in $X$. Then, there exists $\mu, \eta \in L^{X}$ and $\alpha, \beta \in L_{0}$ such that $\mu(x)<\alpha$ $\leq\left(X, \varphi_{1,2}\right.$. int $\left.{ }_{\mu}\right)(y)$ and $(y)<\beta \leq\left(\varphi_{1,2}\right.$.int $\left.\eta_{\eta}\right)(x)$ are hold. This means that $\underset{R_{1}(\rho) \leq \mu}{\bigvee}\left(\boldsymbol{U}\left(R_{1}\right) \wedge \rho(y)\right)>\mu(x)$ and $\underset{R_{2}(\rho) \leq \eta}{\bigvee}\left(\mathcal{U}\left(R_{2}\right) \wedge \rho(x)\right)>\eta(y)$ are also hold for all $\rho \in L^{X}$. Hence, there is $R_{1}, R_{2} L^{X \times X}$ such that $R_{1}(x, y)=$ $\left(X, \varphi_{1,2}\right.$.int $\left.{ }_{\mu}\right)(x)$ if $x=y$ and $R_{1}(x, y)=\mu(x)$ if $x \neq y$ such that $R_{1}(x, y)=0$ and $\boldsymbol{U}\left(R_{1}\right)=1$ and $R_{2}(x, y)=\left(X, \varphi_{1,2}\right.$.int $)(x)$ if $x=y$ and $R_{2}(x, y)=\eta(y)$ if $x \neq y$ such that $R_{2}(x, y)=0$ and $\boldsymbol{U}\left(R_{2}\right)=1$. Thus, in every case $(X, \boldsymbol{U})$ is separated.

\section{Corollary 5.2}

Let $X$ be a non-empty set, $\boldsymbol{U}$ is a fuzzy uniform structure on $X$ and $\varphi_{1}, \varphi_{2} \in O_{\left(L^{X}, \tau_{\mathcal{U}}\right)}$. Then the fuzzy uniform space $(X, \mathcal{U})$ is separated if and only if the associated stratified fuzzy topological space $\left(X, \tau_{u}\right)$ is $F$ $\varphi_{1,2}-T_{1}$ space.

Proof: Immediate from Proposition 5.2 and Theorem 2.2 in AbdAllah [8].

For each fuzzy uniform structure $\boldsymbol{U}$ on the set X, the mapping $h$ : $\mathscr{F}_{L} X \rightarrow \mathscr{F}_{L} X$ which is defined by $h(\mathcal{M})=[\mathcal{M}] \boldsymbol{U}$ for all $\mathcal{M} \in \mathscr{F}_{L} X$ is global homogeneous fuzzy neighborhood structure on $X$ [13]. The mapping $h$ will be called global homogeneous fuzzy neighborhood structure associated to the fuzzy uniform structure $\mathcal{U}_{\text {and }}$ will be denoted by $h_{u}$ 
Citation: Abd-Allah AS, Al-Khedhairi A (2017) The Relations between Characterized Fuzzy Proximity, Fuzzy Compact, Fuzzy Uniform Spaces and Characterized Fuzzy $T_{s}$-Spaces and Fuzzy $R_{k}$-Spaces. J Appl Computat Math 6: 337. doi: 10.4172/2168-9679.1000337

Page 14 of 15

The global fuzzy neighborhood structure $h$ on the set $X$ is said to be symmetric [13], provided that $h(\mathcal{L}) \wedge \mathcal{M}$ exists if and only if $L \wedge h$ $(\mathcal{M})$ exists for all $\mathcal{M}, \mathcal{L} \in \mathcal{F}_{L} X$. As shown in Gähler [13], for each fuzzy uniform structure $\mathcal{U}$, the associated homogenous fuzzy neighborhood structure $h_{u}$ is symmetric and both the global homogenous fuzzy neighborhood structures associated to the fuzzy uniform structures $\mathcal{U}$ and its homogenization $\boldsymbol{U}^{*}$ are coincide.

\section{Proposition 5.3}

Let $f:(X, \mathcal{U}) \rightarrow(Y, \boldsymbol{V})$ be an fuzzy uniformly continuous mapping between fuzzy uniform spaces. Then the mapping $f:\left(X, h_{u}\right) \rightarrow\left(Y, h_{v}\right)$ between the associated global homogeneous fuzzy neighborhood spaces is $\left(h_{\vartheta} h_{\vartheta}\right)$-fuzzy continuous [13].

\section{Proposition 5.4}

Let $f:(X, \mathcal{U}) \rightarrow(Y, \boldsymbol{V})$ be an fuzzy uniformly continuous mapping between fuzzy uniform spaces, $\varphi_{1}, \varphi_{2} \in O_{\left(L^{X}, \tau_{U}\right)}$ and $\psi_{1}, \psi_{2} \in O_{\left(L^{Y}, \tau_{V}\right)}$. Then the mapping $f:\left(X, \varphi_{1,2}\right.$.int $\left._{u}\right) \rightarrow\left(X, \varphi_{1,2}\right.$.int $\left.{ }_{u}\right)$ between the associated characterized fuzzy uniform spaces is $\varphi_{1,2} \psi_{1,2}$-fuzzy continuous.

Proof: Immediate from Proposition 3.3 in Abd-Allah [11] and Proposition 5.3

In the following, we prove that for each fuzzy uniform structure on a set $X$, there is an induced stratified fuzzy proximity on $L^{X}$. Moreover, both the fuzzy uniform structure and this induced stratified fuzzy proximity are associated with the same stratified characterized fuzzy uniform space.

\section{Proposition 5.5}

Let $X$ be a non-empty set, $\boldsymbol{U}_{\text {is a fuzzy uniform structure on } X \text { and }}$ $\varphi_{1}, \varphi_{2} \in O_{\left(X, \tau_{U}\right)}$. Then the binary relation $\delta_{\mathcal{U}}$ on $L^{X}$ which is defined by:

$$
\left(X, \varphi_{1,2} \cdot \text { int }_{\mathcal{u}}\right)=\left(X, \varphi_{1,2} \cdot \text { int }_{\delta_{u}}\right)
$$

for all $\mu, \rho \in L^{X}$ is a stratified fuzzy proximity on $X$. Moreover, both the fuzzy uniform structure $\boldsymbol{U}$ and the induced stratified fuzzy proximity $\delta_{u}$ are associated with the same stratified characterized fuzzy uniform space, that is, $\left(X, \varphi_{1,2} \cdot\right.$ int $\left._{\mathcal{U}}\right)=\left(X, \varphi_{1,2} \cdot\right.$.nt $\left._{\delta_{\mathcal{U}}}\right)$.

Proof: Immediate from (5.2), (5.3) and Proposition 3.2.

\section{Corollary 5.3}

Let $(X, \mathcal{U}),(Y, \mathcal{V})$ are two fuzzy uniform spaces, $\varphi_{1}, \varphi_{2} \in O_{\left(X, \tau_{\delta_{u}}\right)}$ and $\psi_{1}, \psi_{2} \in O_{\left(Y, \tau_{\delta_{v}}\right)}$. Then the mapping $f:(X, \mathcal{U}) \rightarrow(Y, \boldsymbol{U})$ is fuzzy uniformly continuous between fuzzy uniform spaces if and only if the mapping $f:\left(X, \varphi_{1,2} \cdot\right.$.nt $\left._{\delta_{\mathcal{U}}}\right) \rightarrow\left(Y, \psi_{1,2}\right.$. int $\left._{\delta_{v}}\right)$ is $\varphi_{1,2} \psi_{1,2}$-fuzzy continuous between the associated stratified fuzzy proximity spaces.

\section{Proof: Immediate from Propositions 5.4 and 5.5. $\square$}

Because of Propositions 3.7 and 5.5 and Corollary 5.3, we can deduce the result.

\section{Proposition 5.6}

Let $(X, \mathscr{U})$ be an fuzzy uniform space, $F, G \in P(X)$ such that $\mathcal{U}[\dot{F}]=\mathcal{U}\left[\dot{\chi}_{F}\right] \leq \dot{\chi}_{G^{\prime}}=\dot{G}^{\prime}$ and $\varphi_{1}, \varphi_{2} \in O_{\left(X, \tau_{\delta_{U}}\right.}$. If $\Phi$ is the family of all fuzzy uniformly continuous functions $f:(X, \mathcal{U}) \rightarrow\left(I_{L}, \mathcal{U}\right)$ for which $x \in X$ implies $\overline{0} \leq f(x) \leq \overline{1}$, then $\chi_{F}$ and $\chi_{G}$ are $\Phi$-separable.

Proof: Immediate from Propositions 3.7 and 5.5 and Corollary 5.3. Now, we shall prove that the stratified characterized fuzzy uniform space which associated with an fuzzy uniform structure is characterized $F R_{2 \frac{1}{2}}{ }^{-}$space in sense of Abd-Allah et al. [11].

\section{Proposition 5.7}

Let $X$ be a non-empty set, $\boldsymbol{U}$ is a fuzzy uniform structure on $X$ and $\varphi_{1}, \varphi_{2} \in O_{\left(X, \tau_{U}\right)}$. Then the associated stratified characterized fuzzy uniform space $\left(X, \varphi_{1,2}\right.$. int $\left.{ }_{\mathcal{u}}\right)$ with the fuzzy uniform structure $\boldsymbol{U}$ is characterized $F R_{2 \frac{1}{2}}$ - space.

Proof: Let $x X, F \in \varphi_{1,2} C(X)$ such that $x \ddot{\mathrm{I}} F$. Since $\chi_{F^{\prime}}$ is $\varphi_{1,2} \boldsymbol{u}$ -fuzzy neighborhood of $x$, that is, $\mathcal{U}[\dot{x}]=\mathcal{N}_{\varphi_{1,2}}(x) \leq \dot{F}^{\prime}$. On account of Proposition 5.6, we get that $x_{1}$ and $\chi_{F}$ are $\Phi$-separated by the fuzzy uniformly continuous function $f:(X, \mathcal{U}) \rightarrow\left(I_{L}, \mathcal{U}\right)$. Because of Proposition 5.4, the function $f:\left(X, \varphi_{1,2} \cdot\right.$.nt $\left._{\mathcal{u}}\right) \rightarrow\left(I_{L}, \psi_{1,2} \cdot\right.$.nt $\left._{\mathcal{u}^{*}}\right)$ is $\varphi_{1,2} \psi_{1,2}-$ fuzzy continuous. Consequently, $\left(X, \varphi_{1,2} \cdot\right.$.int $\left._{u}\right)$ is characterized $F R_{2 \frac{1}{2}}$ - space.

\section{Corollary 5.4}

Let $(X, \mathcal{U})$ be a separated fuzzy uniform space and $\varphi_{1}, \varphi_{2} \in O_{\left(X, \tau_{\mathcal{U}}\right)}$. Then the associated stratified characterized fuzzy uniform space $\left(X, \varphi_{1,2}\right.$.

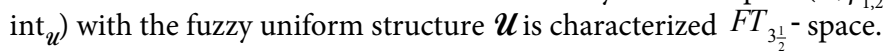

Proof: Immediate from Propositions 5.2 and 5.7.

In the following we give an example of a homogeneous fuzzy uniform structure and we show that the associated stratified characterized fuzzy uniform space is characterized fuzzy uniform $F T_{3 \frac{1}{2}}$ - space.

\section{Example 5.1}

The fuzzy metric in sense of S. Gähler and W. Gähler [24] is canonically generate a homogeneous fuzzy structure as follows: Consider $X$ is non-empty set and $\mathrm{d}$ is a fuzzy metric on $X$, then the mapping $\boldsymbol{U}_{\mathrm{d}}: L^{X \times \mathrm{X}} \rightarrow L$ which is defined by:

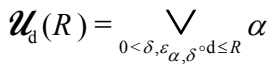

for all $R \in L^{X \times X}$ is a homogeneous fuzzy uniform structures on $X$. Moreover, the associated stratified characterized fuzzy uniform space $\left(X, \varphi_{1,2} \cdot\right.$ int $\left._{u_{\mathrm{d}}}\right)$ is identical with the associated characterized fuzzy metrizable space $\left(X, \varphi_{1,2} \cdot\right.$ int $\left._{\tau_{\mathrm{d}}}\right)$, that is, $\left(X, \varphi_{1,2} \cdot\right.$.nt $\left._{u_{\mathrm{d}}}\right)=\left(X, \varphi_{1,2}\right.$. .nt $\left._{\tau_{\mathrm{d}}}\right)$. Because of Proposition 3.1 in Abd-Allah et al. [22], we have, $\left(X, \varphi_{1,2} \cdot\right.$ int $\left._{\tau_{\mathrm{d}}}\right)$ is characterized $\mathrm{FT}_{4}$-space and therefore $\left(X, \varphi_{1,2}\right.$. int $\left._{\boldsymbol{u}_{\mathrm{i}}}\right)$ is also characterized $\mathrm{FT}_{4}$-space. Hence from Proposition 4.6 in Abd-Allah et al. [11], we get $\left(X, \varphi_{1,2} \cdot\right.$ int $\left._{\mathcal{u}_{\mathrm{d}}}\right)$ is characterized $F T_{3 \frac{1}{2}}$ - space.

\section{Conclusion}

In this paper, we studied the relations between the characterized fuzzy $T_{s}$-spaces, the characterized fuzzy $R_{k}$-spaces presented in Abd-Allah and Abd-Allah and Al-Khedhairi [8-10,11] and the characterized fuzzy proximity spaces presented by Abd-Allah [12], for $s \in\left\{0,1,2,3,3 \frac{1}{2}, 4\right\}$ and $k \in\left\{1,2,2 \frac{1}{2}, 3\right\}$. We also introduced and studied the relations between our characterized fuzzy $T_{s}$-spaces, the characterized fuzzy $R_{k}$-spaces and the characterized fuzzy compact spaces presented by Abd-Allah [12] as a generalization of the weaker and stronger forms to the G-compactness defined by Gähler in 1995. Moreover, we shows here the relations between these characterized fuzzy $T_{s}$-spaces, the characterized fuzzy $R_{k}$-spaces and the characterized fuzzy uniform spaces introduced and studied by Abd-Allah in 2013 as a generalization of the weaker and stronger forms of the fuzzy uniform spaces introduced by Gähler et al. in 1998. 
Citation: Abd-Allah AS, Al-Khedhairi A (2017) The Relations between Characterized Fuzzy Proximity, Fuzzy Compact, Fuzzy Uniform Spaces and Characterized Fuzzy $T_{s}$-Spaces and Fuzzy $R_{k}$-Spaces. J Appl Computat Math 6: 337. doi: 10.4172/2168-9679.1000337

\section{References}

1. Gähler W (1995) The general fuzzy filter approach to fuzzy topology, I. Fuzzy Sets and Systems, 76(2: 205-224.

2. Kasahara S (1979) Operation-compact spaces. Math Japonica 24: 97-105.

3. Abd El-Monsef ME, Zeyada FM, Mashour AS, El-Deeb SN (1983) Operations on the power set $\mathrm{P}(\mathrm{X})$ of a topological space $(\mathrm{X}, \mathrm{T})$ In Colloquium on topology, Janos Bolyai Math. Soc. Eger, Hungry.

4. Kandil A, Abd-Allah AS, Nouh AA (1999) Operations and its applications on L-fuzzy bitopological spaces: Part I. Fuzzy sets and systems 106: 255-274.

5. Abd-Allah AS (2002) General notions related to fuzzy filters. Journal of Fuzzy Mathematics 10: 321-358.

6. Abd-Allah AS, El-Essawy M (2003) On characterizing notions of characterized spaces. Journal of Fuzzy Mathematics 11: 835-876.

7. Abd-Allah AS, El-Essawy M (2004) Closedness and compactness in characterized spaces. Journal of Fuzzy Mathematics 12: 591-632.

8. Abd-Allah AS (2007) Separation axioms in characterized fuzzy spaces. Journal of Fuzzy Mathematics 15: 291. 9. Abd-Allah AS (2008) Fuzzy regularity axioms and $T_{2 \frac{1}{2}}$ axioms in characterized
spaces, J Egypt Math Soci 16: $225-253$.

10. Abd-Allah AS (2010) On characterized fuzzy regular and fuzzy normal spaces. J Egypt Math Soci 18: 9-38.

11. Abd-Allah AS, Al-Khedhairi A (2016) Characterized fuzzy $R_{2 \frac{1}{2}}$ and characterized fuzzy $T_{3 \frac{1}{2}}$-spaces. Indiana Univ Math J pp: 1-31.

12. Abd-Allah AS (2013) Characterized Proximity L-spaces, Characterized
Compact $L$-spaces and Characterized Uniform L-spaces. J Egypt Math Soci 3: 98-119.

13. Gähler W, Bayoumi F, Kandil A, Nouh A (1998) The theory of global fuzzy neighborhood structures (III) Fuzzy uniform structures. Fuzzy Sets and Systems 98: 175-199.

14. Rodabaugh SE, Klement EP, Höhle U (1991) Applications of category theory to fuzzy subsets. Springer Science Business Media 14: 109-136.

15. Bayoumi F, Ibedou I (2004) The relation between GT i-spaces and fuzzy proximity spaces, G-compact spaces, fuzzy uniform spaces. Chaos Solitons Fractals 20: 955-966.

16. Lowen R (1979) Convergence in fuzzy topological spaces. General topology and its applications 10: 147-160.

17. Lowen R (1976) Fuzzy topological spaces and fuzzy compactness. Journal of Mathematical analysis and applications 56: 621-633.

18. Katsaras AK (1980) Fuzzy proximities and fuzzy completely regular spaces. J Anal St Univ Jasi 26: 980.

19. Gähler W, Abd-Allah AS, Kandil A (2000) On extended fuzzy topologies. Fuzzy Sets and Systems 109: 149-172.

20. Chang L (1968) Fuzzy topological spaces. J Math Anal Appl 24: 182-190.

21. Goguen JA (1967) L-fuzzy sets. J Math Anal Appl 18: 145-174.

22. Abd-Allah SA, Al-Khedhairi A (2014) Initial and final characterized $F T_{3 \frac{1}{2}}$ and finer characterized $F R_{2 \frac{1}{2}}$ spaces, Fuzzy Sets and Systems.

23. Katsaras K, Petalas CG (1984) On Fuzzy syntopogenous structures. J Math Anal Appl 99: 219-236

24. Gähler S, Gähler W (1994) Fuzzy real numbers. Fuzzy Sets and Systems 66 137-158. 